\title{
Research Article \\ Optimal Selling Rule in a Regime Switching Lévy Market
}

\author{
Moustapha Pemy \\ Department of Mathematics, Towson University, Towson, MD 21252-0001, USA \\ Correspondence should be addressed to Moustapha Pemy, mpemy@towson.edu
}

Received 5 March 2011; Accepted 5 April 2011

Academic Editor: Giuseppe Marino

Copyright (c) 2011 Moustapha Pemy. This is an open access article distributed under the Creative Commons Attribution License, which permits unrestricted use, distribution, and reproduction in any medium, provided the original work is properly cited.

This paper is concerned with a finite-horizon optimal selling rule problem when the underlying stock price movements are modeled by a Markov switching Lévy process. Assuming that the transaction fee of the selling operation is a function of the underlying stock price, the optimal selling rule can be obtained by solving an optimal stopping problem. The corresponding value function is shown to be the unique viscosity solution to the associated HJB variational inequalities. A numerical example is presented to illustrate the results.

\section{Introduction}

One of the major decision investors have to make on a daily basis is to identify the best time to sell or buy a particular stock. Usually if the right decision is not taken at the right time, this will generally result in large losses for the investor. Such decisions are mainly affected by various macro- and micro-economical parameters. One of the main factors that affect decision making in the marketplace is the trend of the stock market. In this paper, we study trading decision making when we assume that market trends are subject to change and that these fluctuations can be captured by a combination of a latent Markov chain and a jump process. In fact, we model the stock price dynamics with a regime switching Lévy process. Regime switching Lévy processes are obtained by combining a finite number of geometric Lévy processes modulated by a finite-state Markov chain. This type of processes clearly capture the main features of a wide variety of stock such as energy stock and commodities which usually display a lot of spikes and seasonality. Selling rule problems in general have been intensively studied in the literature, and most of the work have been done when the stock price follows a geometric Brownian motion or a simple Markov switching process. Among many others, we can cite the work of Zhang [1]; in this paper, a selling rule is determined by two threshold levels, and a target price and a stop-loss limit are considered. One makes a selling decision whenever the price reaches either the target price or the stop-loss limit. 
The objective is to choose these threshold levels to maximize an expected return function. In [1], such optimal threshold levels are obtained by solving a set of two-point boundary value problems. Recently Pemy and Zhang [2] studied a similar problem in the case where there is no jump process associated and the underlying dynamics is just a traditional Markov switching process built by coupling a set of geometric Brownian motions.

In this paper, we extend the result of Pemy and Zhang [2], we consider an optimal selling rule among the class of almost all stopping times under a regime switching Lévy model. We study the case when the stock has to be sold within a prespecified time limit. Given a transaction cost which is a function of the underlying stock price, the objective is to choose a stopping time so as to maximize an expected return. The optimal stopping problem was studied by McKean [3] back to the 1960s when there is no switching; see also Samuelson [4] in connection with derivative pricing and Øksendal [5] for optimal stopping in general. In models with regime switching, Guo and Zhang [6] considered the model with a two-state Markov chain. Using a smooth-fit technique, they were able to convert the optimal stopping problem to a set of algebraic equations under certain smoothness conditions. Closed-form solutions were obtained in these cases. However, it can be shown with extensive numerical tests that the associated algebraic equations may have no solutions. This suggests that the smoothness $\left(C^{2}\right)$ assumption may not hold in these cases. Moreover, the results in $[5,6]$ are established on an infinite time horizon setup. However, in practice, an investor often has to sell his stock holdings by a certain date due to various nonprice-related considerations such as year-end tax deduction or the need for raising cash for major purchases. In these cases, it is necessary to consider the corresponding optimal selling with a finite horizon. It is the purpose of this paper to treat the underlying finite horizon optimization problem with possible nonsmoothness of the solutions to the associated HJB variational inequalities. We resort to the concept of viscosity solutions and show that the corresponding value function is indeed the only viscosity solution to the HJB variational inequalities. We clearly prove that the value function of this optimal stopping time problem is the unique viscosity solution to the associated $\mathrm{HJB}$ variational inequalities, which enables us to run some numerical schemes in order to approximate the value function and derive the both the continuation region and the stopping region. It is well known that the optimal stopping rule can be determined by the corresponding value function; see, for example, Krylov [7] and Øksendal [5] for diffusions, Pham [8] for jump diffusions, and Guo and Zhang [6] and [9] for regime switching diffusions.

The paper is organized as follows. In the next section, we formulate the problem under consideration and then present the associated HJB inequalities and their viscosity solutions. In Section 3, we obtain the continuity property of the value function and show that it is the only viscosity solution to the HJB equations. In Section 4, we give a numerical example in order to illustrate our results. To better present the results without undue technical difficulties, all proofs are moved to the appendix placed at the end of the paper.

\section{Problem Formulation}

Given an integer $m \geq 2$, let $\alpha(t) \in \mathcal{M}=\{1,2, \ldots, m\}$ denote a Markov chain with an $m \times m$ matrix generator $Q=\left(q_{i j}\right)_{m, m}$, that is, $q_{i j} \geq 0$ for $i \neq j$ and $\Sigma_{j=1}^{m} q_{i j}=0$ for $i \in \mathcal{M}$ and a Lévy process $\left(\eta_{t}\right)_{t}$. Let $N$ be the Poisson random measure of $\left(\eta_{t}\right)_{t}$, then it is defined as follows: for any Borel set $U \subset \mathbb{R}$,

$$
N(t, U)=\sum_{0<s \leq t} \mathbf{1}_{U}\left(\eta_{s}-\eta_{s^{-}}\right)
$$


The differential form of $N$ is denoted by $N(d t, d z)$. Let $v$ be the Lévy measure of $\left(\eta_{t}\right)_{t}$; we have $v(U)=E[N(1, U)]$ for any Borel set $U \subset \mathbb{R}$. We define the differential form $\bar{N}(d t, d z)$ as follows:

$$
\bar{N}(d t, d z)= \begin{cases}N(d t, d z)-v(d z) d t, & \text { if }|z|<1 \\ N(d t, d z), & \text { if }|z| \geq 1\end{cases}
$$

From Lévy-Khintchine formula, we have

$$
\int_{\mathbb{R}} \min \left(|z|^{2}, 1\right) v(d z)<\infty
$$

In our regime switching Lévy market model, the stock price denoted by $X(t)$ satisfies the following Lévy stochastic differential equation

$$
\begin{aligned}
\mathrm{d} X(t) & =X(t)\left(\mu(\alpha(t)) \mathrm{d} t+\sigma(\alpha(t)) \mathrm{d} W(t)+\int_{\mathbb{R}} \gamma(\alpha(t)) z \bar{N}(d t, d z)\right), \\
X(s) & =x, \quad s \leq t \leq T,
\end{aligned}
$$

where $x$ is the initial price and $T$ is a finite time. For each state $i \in \mathcal{M}, \mu(i)$ the rate of return, $\sigma(i)$ the volatility and $\gamma(i)$ the jump intensity are known and satisfied the linear growth condition. There exists a constant $C>0$ such that for all $x \in \mathbb{R}$ and all $t \in[0, T]$, we have

$$
x^{2}\left(\mu(\alpha(t))^{2}+\sigma(\alpha(t))^{2}+\int_{\mathbb{R}}|\gamma(\alpha(t))|^{2} z^{2} v(d z)\right) \leq C\left(1+x^{2}\right)
$$

$W(t)$ is the standard Weiner process, and $\widetilde{N}(d t, d z)$ represents the differential form of the jump measure of $\eta_{t}$. The processes $W(\cdot), \alpha(\cdot)$, and $\eta(\cdot)$ are defined on a probability space $(\Omega, \mathcal{F}, P)$ and are independent of each other.

In this paper, we consider the optimal selling rule with a finite horizon $T$. We assume that the transaction cost function $a(\cdot)>0$ is the function of the stock price itself. In this case, we take into account all costs associated with the selling operation. The main objective of this selling problem is to sell the stock by time $T$ so as to maximize the quantity $E\left[e^{-r(\tau-s)}(X(\tau)-\right.$ $a(X(\tau)))]$, where $r>0$ is a discount rate.

Let $\mathcal{F}_{t}=\sigma\{\alpha(s), W(\mathrm{~s}), \eta(s) ; s \leq t\}$ and let $\Lambda_{s, T}$ denote the set of $\mathcal{F}_{t}$-stopping times such that $s \leq \tau \leq T$ a.s. The value function can be written as follows:

$$
v(s, x, i)=\sup _{\tau \in \Lambda_{s, T}} E\left[e^{-r(\tau-s)}(X(\tau)-a(X(\tau))) \mid X(s)=x, \alpha(s)=i\right] .
$$

Given the value function $v(s, x, i)$, it is typical that an optimal stopping time $\tau^{*}$ can be determined by the following continuation region:

$$
D=\{(t, x, i) \in[0, T) \times \mathbb{R} \times \mathcal{M} ; v(t, x, i)>x-a(x)\},
$$


as follows:

$$
\tau^{*}=\inf \{t>0 ;(t, X(t), \alpha(t)) \notin D\}
$$

It can be proved that if $\tau^{*}<+\infty$, then

$$
v(s, x, i)=E^{s, x, i}\left[e^{-r\left(\tau^{*}-s\right)}\left(X\left(\tau^{*}\right)-a(x)\right)\right] .
$$

Thus, $\tau^{*}$ is the optimal stopping time; see [9].

The process $(X(t), \alpha(t))$ is a Markov process with generator $\mathcal{A}$ defined as follows:

$$
\begin{aligned}
(\mathcal{A} f)(s, x, i)= & \frac{1}{2} x^{2} \sigma^{2}(i) \frac{\partial^{2} f(s, x, i)}{\partial x^{2}}+x \mu(i) \frac{\partial f(s, x, i)}{\partial x}+Q f(s, x, \cdot)(i) \\
& +\int_{\mathbb{R}}\left(f(s, x+\gamma(i) x z)-f(s, x)-\gamma(i) x z \mathbf{1}_{\{|z|<1\}}(z) \frac{\partial f}{\partial x}\right) v(d z),
\end{aligned}
$$

where

$$
Q f(s, x, \cdot)(i)=\sum_{j \neq i} q_{i j}(f(s, x, j)-f(s, x, i)) .
$$

The corresponding Hamiltonian has the following form:

$$
\begin{aligned}
& \mathscr{H}\left(i, s, x, u, \mathrm{D}_{s} u, \mathrm{D}_{x} u, \mathrm{D}_{x}^{2} u\right) \\
& \quad=\min \left[r u(s, x, i)-\frac{\partial u(s, x, i)}{\partial s}-(\mathcal{A} u)(s, x, i), u(s, x, i)-(x-a(x))\right] \\
& \quad=0 .
\end{aligned}
$$

Note that $X(t)>0$ for all $t$. Let $\mathbb{R}^{+}=(0, \infty)$. Formally, the value function $v(s, x, i)$ satisfies the HJB equation

$$
\begin{aligned}
\mathscr{H}\left(i, s, x, v, \mathrm{D}_{s} v, \mathrm{D}_{x} v, \mathrm{D}_{x}^{2} v\right) & =0, \quad \text { for }(s, x, i) \in[0, T) \times \mathbb{R}^{+} \times \mathcal{M}, \\
v(T, x, \alpha(T)) & =(x-a(x)) .
\end{aligned}
$$

In order to study the possibility of existence and uniqueness of a solution of (2.12), we use a notion of viscosity solution introduced by Crandall et al. [10].

Definition 2.1. We say that $f(s, x, i)$ is a viscosity solution of

$$
\begin{aligned}
\mathscr{H}\left(i, s, x, f, \frac{\partial f}{\partial s}, \frac{\partial f}{\partial x}, \frac{\partial^{2} f}{\partial x^{2}}\right) & =0, \quad \text { for } i \in \mathcal{M}, s \in[0, T), x \in \mathbb{R}^{+}, \\
f(T, x, \alpha(T)) & =(x-a(x)) .
\end{aligned}
$$


(1) for all $x \in \mathbb{R}^{+} f(T, x, \alpha(T))=(x-a(x))$, and for each $i \in \mathcal{M}, f(s, x, i)$ is continuous in $(s, x)$, moreover, there exist constants $K$ and $\kappa$ such that

$$
f(s, x, i) \leq K\left(1+|x|^{\kappa}\right),
$$

(2) for each $i \in \mathcal{M}$,

$$
\mathscr{H}\left(i, s_{0}, x_{0}, f, \frac{\partial \phi}{\partial s}, \frac{\partial \phi}{\partial x}, \frac{\partial^{2} \phi}{\partial x^{2}}\right) \leq 0
$$

whenever $\phi(s, x) \in C^{2}$ such that $f(s, x, i)-\phi(s, x)$ has local maximum at $(s, x)=$ $\left(s_{0}, x_{0}\right)$,

(3) and for each $i \in \mathcal{M}$,

$$
\mathscr{H}\left(i, s_{0}, x_{0}, f, \frac{\partial \psi}{\partial s}, \frac{\partial \psi}{\partial x}, \frac{\partial^{2} \psi}{\partial x^{2}}\right) \geq 0
$$

whenever $\psi(s, x) \in C^{2}$ such that $f(s, x, i)-\psi(s, x)$ has local minimum at $(s, x)=$ $\left(s_{0}, x_{0}\right)$.

Let $f$ be a function that satisfies (2.3). It is a viscosity subsolution (resp. supersolution) if it satisfies (2.4) (resp. (2.5)).

\section{Properties of Value Functions}

In this section, we study the continuity of the value function, show that it satisfies the associated HJB equation as a viscosity solution, and establish the uniqueness. We first show the continuity property.

Lemma 3.1. For each $i \in \mathcal{M}$, the value function $v(s, x, i)$ is continuous in $(s, x)$. Moreover, it has at most linear growth rate, that is, there exists a constant $C$ such that $|v(s, x, i)| \leq C(1+|x|)$.

The continuity of the value function and its at most linear growth will be very helpful in deriving the maximum principle which itself guarantees the uniqueness of the value function. The following lemma is a simple version of the dynamic programming principle in optimal stopping. A similar result has been proven in Pemy [9]. For general dynamic programming principle, see Krylov [7] for diffusions, Pham [8] for jump diffusions, and Yin and Zhang [11] for dynamic models with regime switching.

Definition 3.2. For each $\varepsilon>0$, a stopping time $\tau_{\varepsilon} \in \Lambda_{s, T}$ is said to be $\varepsilon$-optimal if

$$
0 \leq v(s, x, i)-E\left[e^{-r\left(\tau_{\varepsilon}-s\right)} v\left(\tau_{\varepsilon}, X\left(\tau_{\varepsilon}\right), \alpha\left(\tau_{\varepsilon}\right)\right)\right] \leq \varepsilon .
$$


Lemma 3.3. (1) Let $\beta, \gamma \in \Lambda_{s, T}$ two stopping time such that $s \leq \beta \leq \gamma$ a.s., then one has

$$
E\left[e^{-r(\beta-s)} v(\beta, X(\beta), \alpha(\beta))\right] \geq E\left[e^{-r(\gamma-s)} v(\gamma, X(\gamma), \alpha(\gamma))\right]
$$

In particular for any stopping time $\theta \in \Lambda_{s, T}$, one has

$$
v(s, x, i) \geq E^{s, x, i}\left[e^{-r(\theta-s)} v(\theta, X(\theta), \alpha(\theta))\right] .
$$

(2) Let $\theta \in \Lambda_{s, T}$ such that $s \leq \theta \leq \tau_{\epsilon}$ for any $\varepsilon>0$, where $\tau_{\epsilon}$ an $\varepsilon$-optimal stopping time. Then, one has

$$
v(s, x, i)=E^{s, x, i}\left[e^{-r(\theta-s)} v(\theta, X(\theta), \alpha(\theta))\right] .
$$

With Lemma 3.3 at our hand, we proceed and show that the value function $v(s, x, i)$ is a viscosity solution of the variational inequality (2.13).

Theorem 3.4. The value function $v(s, x, i)$ is a viscosity solution of (2.13).

\subsection{Uniqueness of the Viscosity Solution}

In this subsection, we will prove that the value function defined in (2.6) is the unique viscosity solution of the HJB equation (2.13). We begin by recalling the definition of parabolic superjet and subjet.

Definition 3.5. Let $f(s, x, i):[0, T] \times \mathbb{R} \times \mathcal{M} \rightarrow \mathbb{R}$. Define the parabolic superjet by

$$
\begin{aligned}
p^{2,+} f(s, x, i)=\{ & (p, q, M) \in \mathbb{R} \times \mathbb{R}: f(t, y, i) \leq f(s, x, i)+p(t-s)+q(y-x) \\
& \left.+\frac{1}{2}(y-x)^{2} M+o\left(|y-x|^{2}\right) \text { as }(t, y) \longrightarrow(s, x)\right\}
\end{aligned}
$$

and its closure is

$$
\begin{aligned}
\bar{p}^{2,+} f(s, x, i)=\left\{(p, q, M)=\lim _{n \rightarrow \infty}\left(p_{n}, q_{n}, M_{n}\right)\right. & \\
& \text { with }\left(p_{n}, q_{n}, M_{n}\right) \in p^{2,+} f\left(s_{n}, x_{n}, i\right) \text { and } \\
& \left.\lim _{n \rightarrow \infty}\left(s_{n}, x_{n}, f\left(s_{n}, x_{n}, i\right)\right)=(x, f(s, x, i))\right\} .
\end{aligned}
$$

Similarly, we define the parabolic subjet $p^{2,-} f(s, x, i)=-p^{2,+}(-f)(s, x, i)$ and its closure $\bar{p}^{2,-} f(s, x, i)=-\bar{p}^{2,+}(-f)(s, x, i)$ 
We have the following result.

Lemma 3.6. $p^{2,+} f(s, x, i)$ (resp. $p^{2,-} f(s, x, i)$ ) consist of the set of $(\partial \phi(s, x) / \partial s, \partial \phi(s, x) /$ $\left.\partial x, \partial^{2} \phi(s, x) / \partial x^{2}\right)$ where $\phi \in \mathcal{C}^{2}([0, T] \times \mathbb{R})$ and $f-\phi$ has a global maximum (resp. minimum) at $(s, x)$.

A proof can be found in Fleming and Soner [12].

The following result from Crandall et al. [10] is crucial for the proof of the uniqueness.

Theorem 3.7 (Crandall et al. [10]). For $i=1,2$, let $\Omega_{i}$ be locally compact subsets of $\mathbb{R}$, and $\Omega=$ $\Omega_{1} \times \Omega_{2}$, and let $u_{i}$ be upper semicontinuous in $[0, T] \times \Omega_{i}$, and $\bar{p}_{\Omega_{i},+}^{2,} u_{i}(t, x)$ the parabolic superjet of $u_{i}(t, x)$, and $\phi$ twice continuously differentiable in a neighborhood of $[0, T] \times \Omega$.

Set

$$
w\left(t, x_{1}, x_{2}\right)=u_{1}\left(t, x_{1}\right)+u_{2}\left(t, x_{2}\right)
$$

for $\left(t, x_{1}, x_{2}\right) \in[0, T] \times \Omega$, and suppose $\left(\widehat{t}, \widehat{x}_{1}, \widehat{x}_{2}\right) \in[0, T] \times \Omega$ is a local maximum of $w-\phi$ relative to $[0, T] \times \Omega$. Moreover, let us assume that there is an $r>0$ such that for every $M>0$ there exists a $C$ such that for $i=1,2$

$$
\begin{gathered}
b_{i} \leq C \text { whenever }\left(b_{i}, q_{i}, X_{i}\right) \in P_{\Omega_{i}}^{2,+} u_{i}\left(t, x_{i}\right), \\
\left|x_{i}-\widehat{x}_{i}\right|+|t-\hat{t}| \leq r, \quad\left|u_{i}\left(t, x_{i}\right)\right|+\left|q_{i}\right|+\left\|X_{i}\right\| \leq M .
\end{gathered}
$$

Then, for each $\epsilon>0$, there exists $X_{i} \in \mathcal{S}(1)=\mathbb{R}$ such that

(1)

$$
\left(b_{i}, D x_{i} \phi(\widehat{t}, \widehat{x}), X_{i}\right) \in \bar{p}_{\Omega_{i}}^{2,+} u_{i}\left(\widehat{t}, \widehat{x}_{i}\right) \quad \text { for } i=1,2,
$$

(2)

$$
-\left(\frac{1}{\epsilon}+\left\|D^{2} \phi(\widehat{x})\right\|\right) I \leq\left(\begin{array}{cc}
X_{1} & 0 \\
0 & X_{2}
\end{array}\right) \leq D^{2} \phi(\widehat{x})+\epsilon\left(D^{2} \phi(\widehat{x})\right)^{2},
$$

(3)

$$
b_{1}+b_{2}=\frac{\partial \phi(\widehat{t}, \widehat{x}, \widehat{y})}{\partial t} .
$$

We have the following maximum principle.

Theorem 3.8 (Comparison Principle). If $v_{1}(t, x, i)$ and $v_{2}(t, x, i)$ are continuous in $(t, x)$ and are, respectively, viscosity subsolution and supersolution of (2.13) with at most a linear growth. Then,

$$
v_{1}(t, x, i) \leq v_{2}(t, x, i) \quad \forall(t, x, i) \in[0, T] \times \mathbb{R}^{+} \times \mathcal{M} .
$$


The following lemma is be very useful in derivation of the maximum principle.

Lemma 3.9. Let $C_{\text {Lip }}([0, T] \times \mathbb{R} \times \mathcal{M})$ be the set of functions $v(s, x, i)$ on $[0, T] \times \mathbb{R} \times \mathcal{M}$ which are continuous with respect to $s$ and Lipschitz continuous with respect to the variable $x$. For fixed $t_{\delta} \in[0, T]$ and $\alpha_{0} \in \mathcal{M}$, let the operator $\mp$ on $\mathbb{R} \times C_{\text {Lip }}([0, T] \times \mathbb{R} \times \mathcal{M}) \times \mathbb{R} \times \mathbb{R}$ be defined as follows:

$$
\begin{aligned}
\mathcal{F}(x, v, \beta, X)= & -\frac{1}{2} x^{2} \sigma^{2}\left(\alpha_{0}\right) X-x \mu\left(\alpha_{0}\right) \beta-Q v\left(t_{\delta}, x, \cdot\right)\left(\alpha_{0}\right) \\
& -\int_{\mathbb{R}}\left(v\left(t_{\delta}, x+\gamma\left(\alpha_{0}\right) x z, \alpha_{0}\right)-v\left(t_{\delta}, x, \alpha_{0}\right)-\gamma\left(\alpha_{0}\right) x z \mathbf{1}_{\{|z|<1\}}(z) \beta\right) v(d z) .
\end{aligned}
$$

Then, there exits a constant $C>0$ such that

$$
\begin{gathered}
\mathcal{F}(y, w, a(x-y)-b y, Y)-\mathcal{F}(x, v, a(x-y)+b x, X) \\
\leq C\left(x^{2} X-y^{2} Y\right)+C a|x-y|^{2} \\
+C b\left(1+\left|x^{2}\right|\right)+Q v\left(t_{\delta}, x, \cdot\right)\left(\alpha_{0}\right)-Q w\left(t_{\delta}, y, \cdot\right)\left(\alpha_{0}\right) \\
+\int_{\mathbb{R}^{n}}\left(v\left(t_{\delta}, x+\gamma\left(\alpha_{0}\right) z x, \alpha_{0}\right)-w\left(t_{\delta}, y+\gamma\left(\alpha_{0}\right) z y, \alpha_{0}\right)\right. \\
\left.-v\left(t_{\delta}, x, \alpha_{0}\right)+w\left(t_{\delta}, y, \alpha_{0}\right)\right) v(d z),
\end{gathered}
$$

for any $v, w \in C_{\text {Lip }}([0, T] \times \mathbb{R} \times \mathcal{M})$ and $x, y, a, b, X, Y \in \mathbb{R}$.

Remark 3.10. Theorem 3.8 obviously implies the uniqueness of the viscosity solution of the variational inequality (2.13). If we assume that (2.13) has two solutions $v_{1}$ and $v_{2}$ with linear growth, then they are both viscosity subsolution and supersolution of (2.13). Therefore, using the fact that $v_{1}$ is subsolution and $v_{2}$ is supersolution, Theorem 3.8 implies that $v_{1}(t, x, i) \leq$ $v_{2}(t, x, i)$ for all $(t, x, i) \in[0, T] \times \mathbb{R}^{+} \times \mathcal{M}$. And conversely, we also have $v_{2}(t, x, i) \leq v_{1}(t, x, i)$ for all $(t, x, i) \in[0, T] \times \mathbb{R}^{+} \times \mathcal{M}$, since $v_{2}$ is subsolution and $v_{1}$ is supersolution. Consequently, we have $v_{1}(t, x, i)=v_{2}(t, x, i)$ for all $(t, x, i) \in[0, T] \times \mathbb{R}^{+} \times \mathcal{M}$, which confirms the fact the value function defined on (2.6) is the unique solution of the variational inequality (2.13).

\section{Numerical Example}

This example is for a stock which share price roughly around \$55 in average; in this example we assume that the market has two main movements: an uptrend and a downtrend. Thus the Markov chain $\alpha$ takes two states $\mathcal{M}=\{1,2\}$, where $\alpha(t)=1$ denotes the uptrend and $\alpha(t)=2$ denotes the downtrend. The transaction fee $a=0.5$, the discount rate $r=0.05$, the return vector is $\mu=(0.01,-0.01)$, the volatility vector is $\sigma=(0.4,0.2)$, the intensity vector is $\lambda=(0.25,0.5)$, the time $T=0.35$ (in year), and the generator of the Markov chain is

$$
Q=\left(\begin{array}{cc}
-0.023 & 0.023 \\
1.023 & -1.023
\end{array}\right) \text {. }
$$




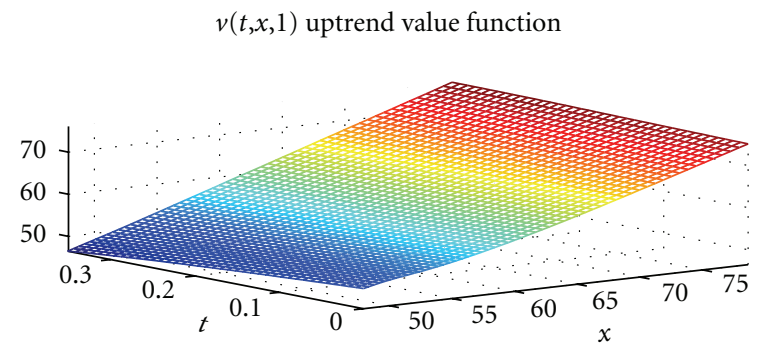

(a)

$v(t, x, 2)$ downtrend value function

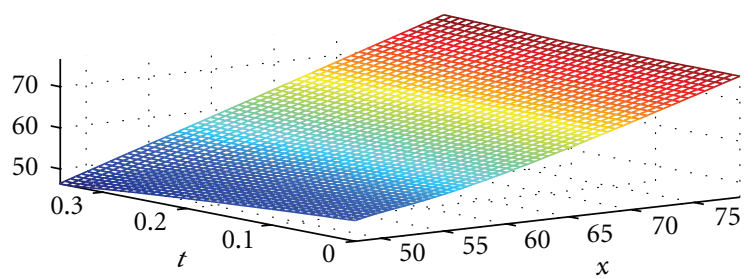

(b)

Figure 1: Value functions $v(t, x, 1)$ for the uptrend and $v(t, x, 2)$ for the downtrend.

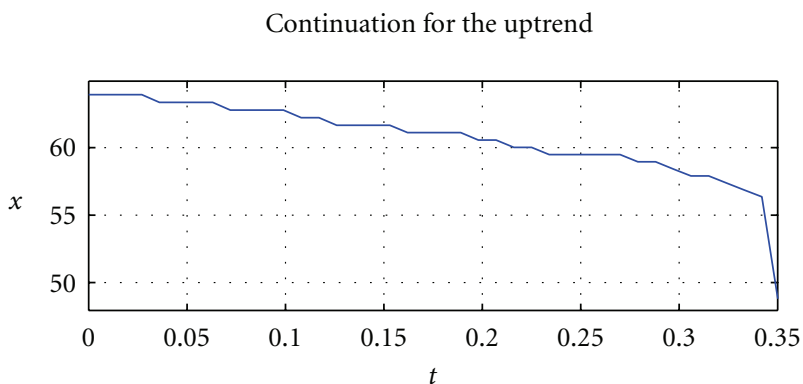

(a)

Continuation for the downtrend

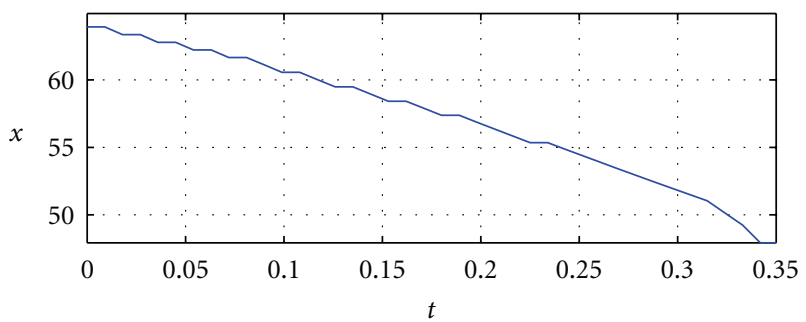

(b)

Figure 2: Free boundary curves.

Figures 1 and 2 represent the value function $v(t, x, i)$ computed by solving the nonlinear system of equations (2.13) and the free boundary curves in both trends. These curves divide the plane in two regions. The region below the free boundary curve is the 
continuation region, and the region above the free boundary curve is the stopping region. In other terms, whenever the share price at any given time within the interval $[0, T]$ is below the free boundary curve, it is optimal for the investor to hold the stock. And whenever the share price is above the free boundary, it is optimal for the investor to sell. This selling rule is obviously easy to implement. This example clearly shows that the selling rule derived by our method can be very attractive to practitioners in an automated trading setting.

\section{Appendix}

\section{A. Proofs of Results}

\section{A.1. Proof of Lemma 3.3}

We have

$$
\begin{aligned}
v(\beta, X(\beta), \alpha(\beta)) & =\sup _{\tau \in \Lambda_{\beta, T}} E\left[e^{-r(\tau-\beta)} g(\alpha(\tau), X(\tau))\right] \\
E\left[e^{-r(\beta-s)} v(\beta, X(\beta), \alpha(\beta))\right] & \geq \sup _{\tau \in \Lambda_{\beta, T}} E\left[e^{-r(\beta-s)} e^{-r(\tau-\beta)} g(\alpha(\tau), X(\tau))\right] \\
& \geq \sup _{\tau \in \Lambda_{\beta, T}} E\left[e^{-r(\tau-s)} g(\alpha(\tau), X(\tau))\right] \\
& \geq \sup _{\tau \in \Lambda_{\gamma, T}} E\left[e^{-r(\gamma-s)} e^{-r(\tau-\gamma)} g(\alpha(\tau), X(\tau))\right] \\
& =E\left[e^{-r(\gamma-s)} v(\gamma, X(\gamma), \alpha(\gamma))\right] .
\end{aligned}
$$

This proves (3.2).

Now let us prove (3.4). Since $\theta \leq \tau_{\epsilon}$ for any $\varepsilon>0$, using (3.2), we have

$$
E\left[e^{-r(\theta-s)} v(\theta, X(\theta), \alpha(\theta))\right] \geq E\left[e^{-r\left(\tau_{\epsilon}-s\right)} v\left(\tau_{\epsilon}, X\left(\tau_{\epsilon}\right), \alpha\left(\tau_{\epsilon}\right)\right)\right]
$$

So using (3.1) and (3.3), we obtain

$$
0 \leq v(s, x, i)-E\left[e^{-r(\theta-s)} v(\theta, X(\theta), \alpha(\theta))\right] \leq v(s, x, i)-E\left[e^{-r\left(\tau_{e}-s\right)} v\left(\tau_{\epsilon}, X\left(\tau_{\epsilon}\right), \alpha\left(\tau_{\epsilon}\right)\right)\right] \leq \varepsilon,
$$

for any $\varepsilon>0$. Thus, letting $\varepsilon \rightarrow 0$, we obtain (3.4). 
International Journal of Mathematics and Mathematical Sciences

\section{A.2. Proof of Lemma 3.1}

Given $x_{1}$ and $x_{2}$, let $X_{1}$ and $X_{2}$ be two solutions of (2.4) with $X_{1}(s)=x_{1}$ and $X_{2}(s)=x_{2}$, respectively. For each $t \in[s, T]$, we have

$$
\begin{aligned}
X_{1}(t)-X_{2}(t)= & x_{1}-x_{2}+\int_{s}^{t}\left(X_{1}(\xi)-X_{2}(\xi)\right) \mu(\alpha(\xi)) d \xi+\int_{s}^{t}\left(X_{1}(\xi)-X_{2}(\xi)\right) \sigma(\alpha(\xi)) d W(\xi) \\
& +\int_{s}^{t} \int_{\mathbb{R}}\left(X_{1}(\xi)-X_{2}(\xi)\right) \gamma(\alpha(\xi)) z \bar{N}(d \xi, d z) .
\end{aligned}
$$

Using the Itô-Lévy isometry, we have

$$
\begin{aligned}
E\left(X_{1}(t)-X_{2}(t)\right)^{2} \leq & C_{0}\left|x_{1}-x_{2}\right|^{2}+C_{1} \int_{s}^{t} E\left(X_{1}(\xi)-X_{2}(\xi)\right)^{2} d \xi+C_{2} \int_{s}^{t} E\left(X_{1}(\xi)-X_{2}(\xi)\right)^{2} d \xi \\
& +C_{3} \int_{s}^{t} \int_{\mathbb{R}} E\left(X_{1}(\xi)-X_{2}(\xi)\right)^{2} z^{2} v(d z) d \xi \\
\leq & C_{0}\left|x_{1}-x_{2}\right|^{2}+\max \left(C_{1}, C_{2}\right) \int_{s}^{t} E\left(X_{1}(\xi)-X_{2}(\xi)\right)^{2} d \xi \\
& +C_{3} \int_{s}^{t} E\left(X_{1}(\xi)-X_{2}(\xi)\right)^{2} d \xi \int_{\mathbb{R}} z^{2} v(d z) .
\end{aligned}
$$

Taking into account (2.5), we can find $K<\infty$ such that $C_{3} \int_{\mathbb{R}} z^{2} v(d z)<K$. The inequality (A.5) becomes

$$
\begin{aligned}
E\left(X_{1}(t)-X_{2}(t)\right)^{2} \leq & C_{0}\left|x_{1}-x_{2}\right|^{2}+\max \left(C_{1}, C_{2}\right) \int_{s}^{t} E\left(X_{1}(\xi)-X_{2}(\xi)\right)^{2} d \xi \\
& +K \int_{s}^{t} E\left(X_{1}(\xi)-X_{2}(\xi)\right)^{2} d \xi .
\end{aligned}
$$

Let $C=\max \left(C_{0}, C_{1}, C_{2}, K\right)$, then (A.7) becomes

$$
E\left(X_{1}(t)-X_{2}(t)\right)^{2} \leq C\left|x_{1}-x_{2}\right|^{2}+C \int_{s}^{t} E\left(X_{1}(\xi)-X_{2}(\xi)\right)^{2} d \xi
$$


Applying Gronwall's inequality, we have

$$
E\left|X_{1}(t)-X_{2}(t)\right|^{2} \leq C\left|x_{1}-x_{2}\right|^{2} e^{C t}
$$

This implies, in view of Cauchy-Schwarz inequality, that

$$
E\left|X_{1}(t)-X_{2}(t)\right| \leq C\left|x_{1}-x_{2}\right| e^{C t}
$$

Using this inequality, we have

$$
\begin{aligned}
v\left(s, x_{1}, i\right)-v\left(s, x_{2}, i\right) & \leq \sup _{\tau \in \Lambda_{s, T}} E\left[e^{-r(\tau-s)}\left|\left(X_{1}(\tau)-a\right)-\left(X_{2}(\tau)-a\right)\right|\right] \\
& \leq \sup _{\tau \in \Lambda_{s, T}} E\left[\left|X_{1}(\tau)-X_{2}(\tau)\right|\right] \\
& \leq C\left|x_{1}-x_{2}\right| e^{C T}
\end{aligned}
$$

This implies the (uniform) continuity of $v(s, x, i)$ with respect to $x$.

We next show the continuity of $v(s, x, i)$ with respect to $s$. Let $X_{t}$ be the solution of (2.4) that starts at $t=s$ with $X(s)=x$ and $\alpha(s)=i$. Let $0 \leq s \leq s^{\prime} \leq T$, we define

$$
\begin{aligned}
X^{\prime}(t) & =X\left(t-\left(s^{\prime}-s\right)\right), \\
\alpha^{\prime}(t) & =\alpha\left(t-\left(s^{\prime}-s\right)\right) .
\end{aligned}
$$

It is easy to show that

$$
E\left(X(t)-X^{\prime}(t)\right)^{2} \leq C\left(s^{\prime}-s\right) \text { for some constant } C>0 .
$$

Given $\tau \in \Lambda_{s, T}$, let $\tau^{\prime}=\tau+\left(s^{\prime}-s\right)$. Then, $\tau^{\prime} \geq s^{\prime}$ and $P\left(\tau^{\prime}>T\right) \rightarrow 0$ as $s^{\prime}-s \rightarrow 0$. that

Let $g(t, x)=e^{-r t}(x-a(x))$. Then, $v(s, x, i)=e^{r s} \sup _{\tau \in \Gamma_{s, T}} E g(\tau, X(\tau))$. It is easy to show

$$
\left|g(s, x)-g\left(s^{\prime}, x^{\prime}\right)\right| \leq\left|x-x^{\prime}\right|+C\left|x^{\prime}-a\left(x^{\prime}\right)\right|\left|s-s^{\prime}\right|,
$$

for some constant $C$.

We define

$$
J(s, x, i, \tau)=e^{r s} \operatorname{Eg}(\tau, X(\tau))
$$


We have

$$
\begin{aligned}
J(s, x, i, \tau) & =e^{r s} \operatorname{Eg}\left(\tau^{\prime}-\left(s^{\prime}-s\right), X^{\prime}\left(\tau^{\prime}\right)\right) \\
& =e^{r s^{\prime}} \operatorname{Eg}\left(\tau^{\prime}, X^{\prime}\left(\tau^{\prime}\right)\right)+o(1) \\
& =e^{r s^{\prime}} \operatorname{Eg}\left(\tau^{\prime}, X^{\prime}\left(\tau^{\prime}\right)\right) I_{\left\{\tau^{\prime} \leq T\right\}}+e^{r s^{\prime}} \operatorname{Eg}\left(\tau^{\prime}, X^{\prime}\left(\tau^{\prime}\right)\right) I_{\left\{\tau^{\prime}>T\right\}}+o(1) \\
& =J\left(s^{\prime}, x, i, \tau^{\prime} \wedge T\right)+o(1),
\end{aligned}
$$

where $o(1) \rightarrow 0$ as $s^{\prime}-s \rightarrow 0$. It follows that

$$
\left|v\left(s^{\prime}, x, i\right)-v(s, x, i)\right| \leq \sup _{\tau \in \Lambda_{s, T}}\left|J\left(s^{\prime}, x, i, \tau^{\prime}\right)-J(s, x, i, \tau)\right| \longrightarrow 0 .
$$

Therefore, we have

$$
\lim _{s^{\prime}-s \rightarrow 0}\left|v\left(s^{\prime}, x, i\right)-v(s, x, i)\right|=0
$$

This gives the continuity of $v$ with respect to $s$.

The joint continuity of $v$ follows from (A.10) and (A.17). Finally, the linear growth inequality follows from (A.10) and

$$
|v(s, x, i)| \leq|x|+|v(s, 0, i)| \leq C(1+|x|) .
$$

This completes the proof.

\section{A.3. Proof of Theorem 3.4}

First we prove that $v(s, x, i)$ is a viscosity supersolution of $(2.13)$. Given $\left(s, x_{s}\right) \in[0, T] \times \mathbb{R}^{+}$, let $\psi \in C^{2}\left([0, T] \times \mathbb{R}^{+}\right)$such that $v(t, x, \alpha)-\psi(t, x)$ has local minimum at $\left(s, x_{s}\right)$ in a neighborhood $N\left(s, x_{s}\right)$. We define a function

$$
\varphi(t, x, i)= \begin{cases}\psi(t, x)+v\left(s, x_{s}, \alpha_{s}\right)-\psi\left(s, x_{s}\right) & \text { if } i=\alpha_{s} \\ v(t, x, i) & \text { if } i \neq \alpha_{s} .\end{cases}
$$


Let $\gamma \geq s$ be the first jump time of $\alpha(\cdot)$ from the initial state $\alpha_{s}$, and let $\theta \in[s, \gamma]$ be such that $(t, X(t))$ starts at $\left(s, x_{s}\right)$ and stays in $N\left(s, x_{s}\right)$ for $s \leq t \leq \theta$. Moreover, $\alpha(t)=\alpha_{s}$, for $s \leq t \leq \theta$. Using Dynkin's formula, we have

$$
\begin{aligned}
& E^{s, x_{s}, \alpha_{s}} e^{-r(\theta-s)} \varphi\left(\theta, X(\theta), \alpha_{s}\right)-\varphi\left(s, x_{s}, \alpha_{s}\right) \\
& =E^{s, x_{s}, \alpha_{s}} \int_{s}^{\theta} e^{-r(t-s)}\left(-r \varphi\left(t, X(t), \alpha_{s}\right)+\frac{\partial \varphi\left(t, X(t), \alpha_{s}\right)}{\partial t}+\frac{1}{2} X_{t}^{2} \sigma^{2}\left(\alpha_{s}\right) \frac{\partial^{2} \varphi\left(t, X(t), \alpha_{s}\right)}{\partial x^{2}}\right. \\
& +X_{t} \mu\left(\alpha_{s}\right) \frac{\partial \varphi\left(t, X(t), \alpha_{s}\right)}{\partial x}+Q \varphi(t, X(t), \cdot)\left(\alpha_{s}\right) \\
& +\int_{\mathbb{R}}\left(\varphi(t, X(t)+\gamma(i) X(t) z)-\varphi(t, X(t))-\gamma(i) X(t) z \mathbf{1}_{\{|z|<1\}}(z) \frac{\partial \varphi}{\partial x}\right) \\
& \times v(d z)) d t
\end{aligned}
$$

Recall that $\left(s, x_{s}\right)$ is the minimum of $v\left(t, x, \alpha_{s}\right)-\psi(t, x)$ in $N\left(s, x_{s}\right)$. For $s \leq t \leq \theta$, we have

$$
v\left(t, X_{t}, \alpha_{s}\right) \geq \psi\left(t, X_{t}\right)+v\left(s, x_{s}, \alpha_{s}\right)-\psi\left(s, x_{s}\right)=\varphi\left(t, X_{t}, \alpha_{s}\right) .
$$

Using (A.19) and (A.21), we have

$$
\begin{aligned}
E^{s, x_{s}, \alpha_{s}} e^{-r(\theta-s)} v\left(\theta, X_{\theta}, \alpha_{s}\right)- & v\left(s, x_{s}, \alpha_{s}\right) \\
\geq E^{s, x_{s}, \alpha_{s}} \int_{s}^{\theta} e^{-r(t-s)}(- & r v\left(t, X(t), \alpha_{s}\right) \\
& +\frac{\partial \psi(t, X(t))}{\partial t}+\frac{1}{2} X_{t}^{2} \sigma^{2}\left(\alpha_{s}\right) \frac{\partial^{2} \psi\left(t, X_{t}\right)}{\partial x^{2}} \\
& +X_{t} \mu\left(\alpha_{s}\right) \frac{\partial \psi\left(t, X_{t}\right)}{\partial x}+Q \varphi\left(t, X_{t}, \cdot\right)\left(\alpha_{s}\right) \\
& \times \int_{\mathbb{R}}\left(\varphi\left(t, X(t)+\gamma(i) X(t) z, \alpha_{s}\right)-\varphi\left(t, X(t), \alpha_{s}\right)\right. \\
& \left.\left.\quad-\gamma(i) X(t) z \mathbf{1}_{\{|z|<1\}}(z) \frac{\partial \psi(t, X(t))}{\partial x}\right) v(d z)\right) d t .
\end{aligned}
$$

Moreover, we have

$$
\begin{aligned}
Q \varphi\left(t, X_{t}, \cdot\right)\left(\alpha_{s}\right) & =\sum_{\beta \neq \alpha_{s}} q_{\alpha_{s} \beta}\left(\varphi\left(t, X_{t}, \beta\right)-\varphi\left(t, X_{t}, \alpha_{s}\right)\right) \\
& \geq \sum_{\beta \neq \alpha_{s}} q_{\alpha_{s} \beta}\left(v\left(t, X_{t}, \beta\right)-v\left(t, X_{t}, \alpha_{s}\right)\right) \\
& \geq Q v\left(t, X_{t}, \cdot\right)\left(\alpha_{s}\right) .
\end{aligned}
$$


Combining (A.22) and (A.23), we have

$$
\begin{aligned}
E^{s, x_{s}, \alpha_{s}} e^{-r(\theta-s)} v\left(\theta, X_{\theta}, \alpha_{s}\right)-v\left(s, x_{s}, \alpha_{s}\right) & \\
\geq E^{s, x_{s}, \alpha_{s}} \int_{s}^{\theta} e^{-r(t-s)}\{- & r v\left(t, X(t), \alpha_{s}\right)+\frac{\partial \psi(t, X(t))}{\partial t}+\frac{1}{2} X_{t}^{2} \sigma^{2}\left(\alpha_{s}\right) \frac{\partial^{2} \psi\left(t, X_{t}\right)}{\partial x^{2}} \\
& +X_{t} \mu\left(\alpha_{s}\right) \frac{\partial \psi\left(t, X_{t}\right)}{\partial x}+Q v\left(t, X_{t}, \cdot\right)\left(\alpha_{s}\right) \\
& +\int_{\mathbb{R}}\left(\varphi\left(t, X(t)+\gamma(i) X(t) z, \alpha_{s}\right)-\varphi\left(t, X(t), \alpha_{s}\right)\right. \\
& \left.\left.-\gamma(i) X(t) z \mathbf{1}_{\{|z|<1\}}(z) \frac{\partial \psi(t, X(t))}{\partial x}\right) v(d z)\right\} d t
\end{aligned}
$$

It follows from Lemma 3.3 that

$$
\begin{aligned}
E^{s, x_{s}, \alpha_{s}} \int_{s}^{\theta} e^{-r(t-s)}( & -r v\left(t, X(t), \alpha_{s}\right)+\frac{\partial \psi(t, X(t))}{\partial t}+\frac{1}{2} X^{2}(t) \sigma^{2}\left(\alpha_{s}\right) \frac{\partial^{2} \psi(t, X(t))}{\partial x^{2}} \\
+ & X(t) \mu\left(\alpha_{s}\right) \frac{\partial \psi(t, X(t))}{\partial x}+Q v\left(t, X_{t}, \cdot\right)\left(\alpha_{s}\right) \\
+ & \int_{\mathbb{R}}\left(\varphi\left(t, X(t)+\gamma(i) X(t) z, \alpha_{s}\right)-\varphi\left(t, X(t), \alpha_{s}\right)\right. \\
& \left.\left.-\gamma(i) X(t) z \mathbf{1}_{\{|z|<1\}}(z) \frac{\partial \psi(t, X(t))}{\partial x}\right) v(d z)\right) d t \leq 0 .
\end{aligned}
$$

Dividing both sides by $E \theta>0$ and sending $\theta \rightarrow s$ lead to

$$
\begin{gathered}
-r v\left(s, x_{s}, \alpha_{s}\right)+\frac{\partial \psi\left(s, x_{s}\right)}{\partial t}+\frac{1}{2} x_{s}^{2} \sigma^{2}\left(\alpha_{s}\right) \frac{\partial^{2} \psi\left(s, x_{s}\right)}{\partial x^{2}}+x_{s} \mu\left(\alpha_{s}\right) \frac{\partial \psi\left(s, x_{s}\right)}{\partial x}+Q v\left(s, x_{s}, \cdot\right)\left(\alpha_{s}\right) \\
+\int_{\mathbb{R}}\left(v\left(s, x_{s}+\gamma(i) x_{s} z, \alpha_{s}\right)-v\left(s, x_{s}, \alpha_{s}\right)-\gamma(i) x_{s} z \mathbf{1}_{\{|z|<1\}}(z) \frac{\partial \psi\left(s, x_{s}\right)}{\partial x}\right) v(d z) \leq 0 .
\end{gathered}
$$

By definition, $v(s, x, i) \geq x-a(x)$. The supersolution inequality follows from this inequality and (A.27).

Now, let us prove the subsolution inequality, namely, that let $\phi \in \mathcal{C}^{1,2}\left([s, T] \times \mathbb{R}^{+}\right)$and $v\left(t, x, \alpha_{s}\right)-\phi(t, x)$ has a local maximum at $\left(s, x_{s}\right) \in[s, T] \times \mathbb{R}^{+}$, then we can assume without loss of generality that $v\left(s, x_{s}, \alpha_{s}\right)-\phi\left(s, x_{s}\right)=0$.

Define

$$
\Phi(t, x, i)= \begin{cases}\phi(t, x) & \text { if } i=\alpha_{s} \\ v(t, x, i) & \text { if } i \neq \alpha_{s}\end{cases}
$$


Let $\gamma$ be the first jump time of $\alpha(\cdot)$ from the state $\alpha_{s}$, and let $\theta_{0} \in[s, \gamma]$ be such that $(t, X(t))$ starts at $\left(s, x_{s}\right)$ and stays in $N\left(s, x_{s}\right)$ for $s \leq t \leq \theta_{0}$. Since $\theta_{0} \leq \gamma$ we have $\alpha(t)=\alpha_{s}$, for $s \leq t \leq \theta_{0}$, and let $\tau_{D}$ be the optimal stopping time, and for $s \leq \theta \leq \min \left(\tau_{D}, \theta_{0}\right)$, we have from Lemma 3.3 (The appendix)

$$
v\left(s, x_{s}, \alpha_{s}\right) \leq E^{s, x_{s}, \alpha_{s}}\left[e^{-r(\theta-s)} v(\theta, X(\theta), \alpha(\theta))\right]
$$

Moreover, since $v\left(s, x_{s}, \alpha_{s}\right)-\phi\left(s, x_{s}\right)=0$ and attains its maximum at $\left(s, x_{s}\right)$ in $N\left(s, x_{s}\right)$, then

$$
v(\theta, X(\theta), \alpha(\theta)) \leq \phi(\theta, X(\theta)) .
$$

Thus, we also have

$$
v(\theta, X(\theta), \alpha(\theta)) \leq \Phi(\theta, X(\theta), \alpha(\theta)) .
$$

This implies, using Dynkin's formula, that

$$
\begin{aligned}
& E^{s, x_{s}, \alpha_{s}} e^{-r(\theta-s)} v\left(\theta, X(\theta), \alpha_{s}\right) \\
& \leq E^{s, x_{s}, \alpha_{s}} e^{-r(\theta-s)} \Phi\left(\theta, X(\theta), \alpha_{s}\right) \\
&=\Phi\left(s, x_{s}, \alpha_{s}\right)+E^{s, x_{s}, \alpha_{s}} \int_{s}^{\theta} e^{-r(t-s)}\left[\frac{\partial \phi(t, X(t))}{\partial t}-r \Phi(t, X(t), \alpha(t))\right. \\
&+ X(t) \mu\left(\alpha_{s}\right) \frac{\partial \phi(t, X(t))}{\partial x}+Q \Phi(t, X(t), \cdot)\left(\alpha_{s}\right) \\
&+\frac{1}{2} X(t)^{2} \sigma^{2}\left(\alpha_{s}\right) \frac{\partial^{2} \phi(t, X(t))}{\partial x^{2}} \\
&+\int_{\mathbb{R}}\left(\Phi\left(t, X(t)+\gamma(i) X(t) z, \alpha_{s}\right)-\Phi\left(t, X(t), \alpha_{s}\right)\right. \\
&\left.\left.-\gamma(i) X(t) z \mathbf{1}_{\{|z|<1\}}(z) \frac{\partial \phi(t, X(t))}{\partial x}\right) v(d z)\right] d t .
\end{aligned}
$$

Note that

$$
\begin{aligned}
Q \Phi(t, X(t), \cdot)\left(\alpha_{s}\right) & =\sum_{\beta \neq \alpha_{s}} q_{\alpha_{s} \beta}(v(t, X(t), \beta)-\phi(t, X(t))) \\
& \leq \sum_{\beta \neq \alpha_{s}} q_{\alpha_{s} \beta}\left(v(t, X(t), \beta)-v\left(t, X(t), \alpha_{s}\right)\right) \\
& \leq Q v(t, X(t), \cdot)\left(\alpha_{s}\right) .
\end{aligned}
$$


Using (A.27) and (A.32), we obtain

$$
\begin{aligned}
& E^{s, x_{s}, \alpha_{s}} e^{-r(\theta-s)} v\left(\theta, X(\theta), \alpha_{s}\right) \\
& \leq E^{s, x_{s}, \alpha_{s}} e^{-r \theta-s} \Phi\left(\theta, X(\theta), \alpha_{s}\right) \\
&=\phi\left(s, x_{s}\right)+E^{s, x_{s}, \alpha_{s}} \int_{s}^{\theta} e^{-r(t-s)}\left[\frac{\partial \phi(t, X(t))}{\partial t}+X(t) \mu\left(\alpha_{s}\right) \frac{\partial \phi(t, X(t))}{\partial x}-r v\left(t, X(t), \alpha_{s}\right)\right. \\
&+ \frac{1}{2} X(t)^{2} \sigma^{2}\left(\alpha_{s}\right) \frac{\partial^{2} \phi(t, X(t))}{\partial x^{2}}+Q v(t, X(t), \cdot)\left(\alpha_{s}\right) \\
&+\int_{\mathbb{R}}\left(\Phi\left(t, X(t)+\gamma(i) X(t) z, \alpha_{s}\right)-\Phi\left(t, X(t), \alpha_{s}\right)\right. \\
&\left.\left.-\gamma(i) X(t) z \mathbf{1}_{\{|z|<1\}}(z) \frac{\partial \phi(t, X(t))}{\partial x}\right) v(d z)\right] d t .
\end{aligned}
$$

Recall that, $v\left(s, x_{s}, \alpha_{s}\right)=\phi\left(s, x_{s}\right)$ by assumption. From (A.28), we deduce

$$
\begin{aligned}
0 \leq E^{, x_{s}, \alpha_{s}} e^{-r(\theta-s)} v\left(\theta, X(\theta), \alpha_{s}\right)-\phi\left(s, x_{s}\right) \\
\leq E^{s, x_{s}, \alpha_{s}} \int_{s}^{\theta} e^{-r t}\left[-r v\left(t, X(t), \alpha_{s}\right)+\frac{\partial \phi(t, X(t))}{\partial t}+\frac{1}{2} X(t)^{2} \sigma^{2}\left(\alpha_{s}\right) \frac{\partial^{2} \phi\left(X(t), \alpha_{s}\right)}{\partial x^{2}}\right. \\
+X(t) \mu\left(\alpha_{s}\right) \frac{\partial \phi\left(X(t), \alpha_{s}\right)}{\partial x}+Q v(t, X(t), \cdot)\left(\alpha_{s}\right) \\
+\int_{\mathbb{R}}\left(\Phi\left(t, X(t)+\gamma(i) X(t) z, \alpha_{s}\right)\right. \\
\left.\left.\quad-\Phi\left(t, X(t), \alpha_{s}\right)-\gamma(i) X(t) z \mathbf{1}_{\{|z|<1\}}(z) \frac{\partial \phi(t, X(t))}{\partial x}\right) v(d z)\right] d t
\end{aligned}
$$

Dividing the last inequality by $E \theta>0$ and sending $\theta \downarrow s$ give

$$
\begin{array}{r}
r v\left(s, x_{s}, \alpha_{s}\right)-\frac{\partial \phi\left(s, x_{s}\right)}{\partial t}-\frac{1}{2} x_{s}^{2} \sigma^{2}\left(\alpha_{s}\right) \frac{\partial^{2} \phi\left(x_{s}, \alpha_{s}\right)}{\partial x^{2}}-x_{s} \mu\left(\alpha_{s}\right) \frac{\partial \phi\left(x_{s}, \alpha_{s}\right)}{\partial x}-Q v\left(s, x_{s}, \cdot\right)\left(\alpha_{s}\right) \\
-\int_{\mathbb{R}}\left(v\left(s, x_{s}+\gamma(i) x_{s} z, \alpha_{s}\right)-v\left(s, x_{s}, \alpha_{s}\right)-\gamma(i) x_{s} z \mathbf{1}_{\{|z|<1\}}(z) \frac{\partial \phi\left(s, x_{s}\right)}{\partial x}\right) v(d z) \leq 0 .
\end{array}
$$

This gives the subsolution inequality. Therefore, $v(t, x, \alpha)$ is a viscosity solution of (2.13). 


\section{A.4. Proof of Lemma 3.9}

Let $v, w \in C_{\operatorname{Lip}}([0, T] \times \mathbb{R} \times \mathcal{M})$ and $x, y, \lambda, a, b, X, Y \in \mathbb{R}$, then we have

$$
\begin{aligned}
\mathcal{F} & (y, w, a(x-y)-b y, Y)-\mathcal{F}(x, v, a(x-y)+b x, X) \\
= & -\frac{1}{2} y^{2} \sigma^{2}\left(\alpha_{0}\right) Y-y \mu\left(\alpha_{0}\right)(a(x-y)-b y)-Q w\left(t_{\delta}, y, \cdot\right)\left(\alpha_{0}\right) \\
& -\int_{\mathbb{R}}\left(w\left(t_{\delta}, y+\gamma\left(\alpha_{0}\right) y z, \alpha_{0}\right)-w\left(t_{\delta}, y, \alpha_{0}\right)-\gamma\left(\alpha_{0}\right) y z \mathbf{1}_{\{|z|<1\}}(z)[a(x-y)-b y]\right) v(d z) \\
& +\frac{1}{2} x^{2} \sigma^{2}\left(\alpha_{0}\right) X+x \mu\left(\alpha_{0}\right)(a(x-y)+b x)+Q v\left(t_{\delta}, x, \cdot\right)\left(\alpha_{0}\right) \\
& +\int_{\mathbb{R}}\left(v\left(t_{\delta}, x+\gamma\left(\alpha_{0}\right) x z, \alpha_{0}\right)-v\left(t_{\delta}, x, \alpha_{0}\right)-\gamma\left(\alpha_{0}\right) x z \mathbf{1}_{\{|z|<1\}}(z)[a(x-y)+b x]\right) v(d z) \\
= & \frac{1}{2} \sigma^{2}\left(\alpha_{0}\right)\left(x^{2} X-y^{2} Y\right)+\mu\left(\alpha_{0}\right)\left(a(x-y)^{2}+b\left(x^{2}+y^{2}\right)\right)+Q v\left(t_{\delta}, x, \cdot\right)\left(\alpha_{0}\right)-Q w\left(t_{\delta}, y, \cdot\right)\left(\alpha_{0}\right) \\
& +\int_{\mathbb{R}}\left(\left[v\left(t_{\delta}, x+\gamma\left(\alpha_{0}\right) x z, \alpha_{0}\right)-w\left(t_{\delta}, y+\gamma\left(\alpha_{0}\right) y z, \alpha_{0}\right)\right]\right. \\
& \left.\quad-\left[v\left(t_{\delta}, x, \alpha_{0}\right)-w\left(t_{\delta}, y, \alpha_{0}\right)\right]-z \gamma\left(\alpha_{0}\right) \mathbf{1}_{\{|z|<1\}}(z)\left[a(x-y)^{2}+b\left(x^{2}+y^{2}\right)\right]\right) v(d z) \\
\leq & \mu\left(\alpha_{0}\right) a|x-y|^{2}+\mu\left(\alpha_{0}\right) b\left(x^{2}+y^{2}\right)+\frac{1}{2} \sigma^{2}\left(\alpha_{0}\right)\left(x^{2} X-y^{2} Y\right)+Q v\left(t_{\delta}, x, \cdot\right)\left(\alpha_{0}\right)-Q w\left(t_{\delta}, y, \cdot\right)\left(\alpha_{0}\right) \\
& +\int_{\mathbb{R}}\left(\left[v\left(t_{\delta}, x+\gamma\left(\alpha_{0}\right) x z, \alpha_{0}\right)-w\left(t_{\delta}, y+\gamma\left(\alpha_{0}\right) y z, \alpha_{0}\right)\right]\right. \\
& \left.\quad-\left[v\left(t_{\delta}, x, \alpha_{0}\right)-w\left(t_{\delta}, y, \alpha_{0}\right)\right]+\left|z \gamma\left(\alpha_{0}\right) \mathbf{1}_{\{|z|<1\}}(z)\right|\left[\left|a(x-y)^{2}\right|+\left|b\left(x^{2}+y^{2}\right)\right|\right]\right) v(d z) .
\end{aligned}
$$

Note that from the Lévy-Khintchine inequality (2.3), one can prove $\int_{\{|z|<1\}}|z| v(d z)<\infty$; therefore, there exists a constant $C>0$ such that

$$
\begin{aligned}
& \mathcal{F}(y, w, a(x-y)-b y, Y)-\mathcal{F}(x, v, a(x-y)+b x, X) \\
& \leq C\left(a|x-y|^{2}+b\left(x^{2}+y^{2}\right)+\left(x^{2} X-y^{2} Y\right)\right)+Q v\left(t_{\delta}, x, \cdot\right)\left(\alpha_{0}\right)-Q w\left(t_{\delta}, y, \cdot\right)\left(\alpha_{0}\right) \\
& \quad+\int_{\mathbb{R}}\left(\left[v\left(t_{\delta}, x+\gamma\left(\alpha_{0}\right) x z, \alpha_{0}\right)-w\left(t_{\delta}, y+\gamma\left(\alpha_{0}\right) y z, \alpha_{0}\right)\right]-\left[v\left(t_{\delta}, x, \alpha_{0}\right)-w\left(t_{\delta}, y, \alpha_{0}\right)\right]\right) v(d z) .
\end{aligned}
$$

This proves (3.14).

Now, let us proof the theorem. 


\section{A.5. Proof of Theorem 3.8}

For any $0<\delta<1$ and $0<\eta<1$, we define

$$
\begin{aligned}
\Phi(t, x, y, i) & =v_{1}(t, x, i)-v_{2}(t, y, i)-\frac{1}{\delta}|x-y|^{2}-\eta e^{(T-t)}\left(x^{2}+y^{2}\right) \\
\phi(t, x, y) & =\frac{1}{\delta}|x-y|^{2}+\eta e^{(T-t)}\left(x^{2}+y^{2}\right) .
\end{aligned}
$$

Note that $v_{1}(t, x, i)$ and $v_{2}(t, x, i)$ satisfy the linear growth. Then, we have for each $i \in \mathcal{M}$

$$
\lim _{|x|+|y| \rightarrow \infty} \Phi(t, x, y, i)=-\infty
$$

and since $\Phi$ is a continuous in $(t, x, y)$, therefore it has a global maximum at a point $\left(t_{\delta}, x_{\delta}, y_{\delta}, \alpha_{0}\right)$. Observe that

$$
\Phi\left(t_{\delta}, x_{\delta}, x_{\delta}, \alpha_{0}\right)+\Phi\left(t_{\delta}, y_{\delta}, y_{\delta}, \alpha_{0}\right) \leq 2 \Phi\left(t_{\delta}, x_{\delta}, y_{\delta}, \alpha_{0}\right)
$$

It implies

$$
\begin{aligned}
v_{1}\left(t_{\delta}, x_{\delta}, \alpha_{0}\right) & -v_{2}\left(t_{\delta}, x_{\delta}, \alpha_{0}\right)-2 \eta e^{\left(T-t_{\delta}\right)}\left(x_{\delta}^{2}\right)+v_{1}\left(t_{\delta}, y_{\delta}, \alpha_{0}\right)-v_{2}\left(t_{\delta}, y_{\delta}, \alpha_{0}\right)-2 \eta e^{\left(T-t_{\delta}\right)}\left(y_{\delta}^{2}\right) \\
& \leq 2 v_{1}\left(t_{\delta}, x_{\delta}, \alpha_{0}\right)-2 v_{2}\left(t_{\delta}, y_{\delta}, \alpha_{0}\right)-\frac{2}{\delta}\left|x_{\delta}-y_{\delta}\right|^{2}-2 \eta e^{\left(T-t_{\delta}\right)}\left(x_{\delta}^{2}+y_{\delta}^{2}\right) .
\end{aligned}
$$

Then,

$$
\begin{aligned}
& -v_{2}\left(t_{\delta}, y_{\delta}, \alpha_{0}\right)-2 e^{\left(T-t_{\delta}\right)} \eta\left(x_{\delta}^{2}\right)+v_{1}\left(t_{\delta}, x_{\delta}, \alpha_{0}\right)-2 \eta e^{\left(T-t_{\delta}\right)}\left(y_{\delta}^{2}\right) \\
& \leq v_{1}\left(t_{\delta}, x_{\delta}, \alpha_{0}\right)-v_{2}\left(t_{\delta}, y_{\delta}, \alpha_{0}\right)-\frac{2}{\delta}\left|x_{\delta}-y_{\delta}\right|^{2}-2 \eta e^{\left(T-t_{\delta}\right)}\left(x_{\delta}^{2}+y_{\delta}^{2}\right) .
\end{aligned}
$$

Consequently, we have

$$
\frac{2}{\delta}\left|x_{\delta}-y_{\delta}\right|^{2} \leq\left(v_{1}\left(t_{\delta}, x_{\delta}, \alpha_{0}\right)-v_{1}\left(t_{\delta}, y_{\delta}, \alpha_{0}\right)\right)+\left(v_{2}\left(t_{\delta}, x_{\delta}, \alpha_{0}\right)-v_{2}\left(t_{\delta}, y_{\delta}, \alpha_{0}\right)\right)
$$

By the linear growth condition, we know that there exist $K_{1}, K_{2}$ such that $v_{1}(t, x, i) \leq K_{1}(1+$ $|x|)$ and $v_{2}(t, x, i) \leq K_{2}(1+|x|)$. Therefore, there exists $C$ such that we have

$$
\frac{2}{\delta}\left|x_{\delta}-y_{\delta}\right|^{2} \leq C\left(1+\left|x_{\delta}\right|+\left|y_{\delta}\right|\right)
$$


So,

$$
\left|x_{\delta 1}^{0}-x_{\delta 2}^{0}\right|^{2} \leq \delta C\left(1+\left|x_{\delta 1}^{0}\right|^{\kappa_{1}}+\left|x_{\delta 1}^{0}\right|^{\kappa_{2}}\right)
$$

We also have $\Phi\left(s, 0,0, \alpha_{0}\right) \leq \Phi\left(t_{\delta}, x_{\delta}, y_{\delta}, \alpha_{0}\right)$ and $\left|\Phi\left(s, 0,0, \alpha_{0}\right)\right| \leq K\left(1+\left|x_{\delta}\right|+\left|y_{\delta}\right|\right)$. This leads to

$$
\begin{aligned}
\eta e^{\left(-t_{\delta}\right)}\left(x_{\delta}^{2}+y_{\delta}^{2}\right) & \leq v_{1}\left(t_{\delta}, x_{\delta}, \alpha_{0}\right)-v_{2}\left(t_{\delta}, y_{\delta}, \alpha_{0}\right)-\frac{1}{\delta}\left|x_{\delta}-y_{\delta}\right|^{2}-\Phi\left(s, 0,0, \alpha_{0}\right) \\
& \leq 3 C\left(1+\left|x_{\delta}\right|+\left|y_{\delta}\right|\right) .
\end{aligned}
$$

It comes that

$$
\frac{\eta e^{\left(T-t_{\delta}\right)}\left(x_{\delta}^{2}+y_{\delta}^{2}\right)}{\left(1+\left|x_{\delta}\right|+\left|y_{\delta}\right|\right)} \leq 3 C
$$

therefore there exists $C_{\eta}$ such that

$$
\left|x_{\delta}\right|+\left|y_{\delta}\right| \leq C_{\eta}, \quad t_{\delta} \in[s, T]
$$

The inequality (A.48) implies the sets $\left\{x_{\delta}, \delta>0\right\}$ and $\left\{y_{\delta}, \delta>0\right\}$ are bounded by $C_{\eta}$ independent of $\delta$, so we can extract convergent subsequences that we also denote $\left(x_{\delta}\right)_{\delta},\left(y_{\delta}\right)_{\delta^{\prime}}$ and $\left(t_{\delta}\right)_{\delta}$. Moreover, from the inequality (A.45), it comes that the exists $x_{0}$ such that

$$
\lim _{\delta \rightarrow 0} x_{\delta}=x_{0}=\lim _{\delta \rightarrow 0} y_{\delta}, \quad \lim _{\delta \rightarrow 0} t_{\delta}=t_{0} .
$$

Using (A.43) and the previous limit, we obtain

$$
\lim _{\delta \rightarrow 0} \frac{2}{\delta}\left|x_{\delta}-y_{\delta}\right|^{2}=0
$$

$\Phi$ achieves its maximum at $\left(t_{\delta}, x_{\delta}, y_{\delta}, \alpha_{0}\right)$, so by the Theorem 3.7 for each $\epsilon>0$, there exist $b_{1 \delta}, b_{2 \delta}, X_{\delta}$, and $Y_{\delta}$ such that

$$
\begin{gathered}
\left(b_{1 \delta}, \frac{2}{\delta}\left(x_{\delta}-y_{\delta}\right)+2 \eta e^{(T-t)} x_{\delta}, X_{\delta}\right) \in \bar{D}^{2,+} v_{1}\left(t_{\delta}, x_{\delta}, \alpha_{0}\right) \\
\left(-b_{2 \delta},-\frac{2}{\delta}\left(x_{\delta}-y_{\delta}\right)+2 \eta e^{(T-t)} y_{\delta},-Y_{\delta}\right) \in \bar{D}^{2,+}\left(-v_{2}\left(t_{\delta}, y_{\delta}, \alpha_{0}\right)\right)
\end{gathered}
$$

But, we know that

$$
\bar{p}^{2,+}\left(-v_{2}\left(t_{\delta}, y_{\delta}, \alpha_{0}\right)\right)=-\bar{p}^{2,-} v_{2}\left(t_{\delta}, y_{\delta}, \alpha_{0}\right)
$$


Therefore, we obtain

$$
\left(b_{2 \delta}, \frac{2}{\delta}\left(x_{\delta}-y_{\delta}\right)-2 \eta e^{(T-t)} y_{\delta}, Y_{\delta}\right) \in \bar{p}^{2,-} v_{2}\left(t_{\delta}, y_{\delta}, \alpha_{0}\right)
$$

Equation (A.51) implies by the definition of the viscosity solution that

$$
\begin{gathered}
\min \left[r v_{1}\left(t_{\delta}, x_{\delta}, \alpha_{0}\right)-b_{1 \delta}-\frac{1}{2} x_{\delta}^{2} \sigma^{2}\left(\alpha_{0}\right) X_{\delta}-x_{\delta} \mu\left(\alpha_{0}\right)\left(\frac{2}{\delta}\left(x_{\delta}-y_{\delta}\right)+2 \eta e^{(T-t)} x_{\delta}\right)-Q v_{1}\left(t_{\delta}, x_{\delta}, \cdot\right)\left(\alpha_{0}\right)\right. \\
-\int_{\mathbb{R}}\left(v_{1}\left(t_{\delta}, x_{\delta}+r\left(\alpha_{0}\right) x_{\delta} z, \alpha_{0}\right)-v_{1}\left(t_{\delta}, x_{\delta}, \alpha_{0}\right)-r\left(\alpha_{0}\right) x_{\delta} z \mathbf{1}_{\{|z|<1\}}(z)\right. \\
\left.\left.\quad \times\left(\frac{2}{\delta}\left(x_{\delta}-y_{\delta}\right)+2 \eta e^{(T-t)} x_{\delta}\right)\right) v(d z), v_{1}\left(t_{\delta}, x_{\delta}, \alpha_{0}\right)-\left(x_{\delta}-a\right)\right] \leq 0 .
\end{gathered}
$$

Consequently, we have two cases; either

$$
v_{1}\left(t_{\delta}, x_{\delta}, \alpha_{0}\right)-\left(x_{\delta}-a\right) \leq 0
$$

or

$$
\begin{gathered}
r v_{1}\left(t_{\delta}, x_{\delta}, \alpha_{0}\right)-b_{1 \delta}-\frac{1}{2} x_{\delta}^{2} \sigma^{2}\left(\alpha_{0}\right) X_{\delta}-x_{\delta} \mu\left(\alpha_{0}\right)\left(\frac{2}{\delta}\left(x_{\delta}-y_{\delta}\right)+2 \eta e^{(T-t)} x_{\delta}\right)-Q f\left(s, x_{\delta}, \cdot\right)\left(\alpha_{0}\right) \\
-\int_{\mathbb{R}}\left(v_{1}\left(t_{\delta}, x_{\delta}+\gamma\left(\alpha_{0}\right) x_{\delta} z\right)-v_{1}\left(t_{\delta}, x_{\delta}\right)\right. \\
\left.\quad-\gamma\left(\alpha_{0}\right) x_{\delta} z \mathbf{1}_{\{|z|<1\}}(z) \times\left(\frac{2}{\delta}\left(x_{\delta}-y_{\delta}\right)+2 \eta e^{(T-t)} x_{\delta}\right)\right) v(d z) \leq 0 .
\end{gathered}
$$

First of all, we assume that $v_{1}\left(t_{\delta}, x_{\delta}, \alpha_{0}\right)-\left(x_{\delta}-a\right) \leq 0$. And similarly, (A.54) implies by the definition of the viscosity solution that

$$
\begin{aligned}
\min \left[r v_{2}\left(t_{\delta}, y_{\delta}, \alpha_{0}\right)-b_{2 \delta}-\frac{1}{2} y_{\delta}^{2} \sigma^{2}\left(\alpha_{0}\right) Y_{\delta}-y_{\delta} \mu\left(\alpha_{0}\right)\left(\frac{2}{\delta}\left(x_{\delta}-y_{\delta}\right)-2 \eta e^{(T-t)} y_{\delta}\right)-Q v_{2}\left(t_{\delta}, y_{\delta}, \cdot\right)\left(\alpha_{0}\right)\right. \\
-\int_{\mathbb{R}}\left(v_{2}\left(t_{\delta}, y_{\delta}+\gamma\left(\alpha_{0}\right) y_{\delta} z, \alpha_{0}\right)-v_{2}\left(t_{\delta}, y_{\delta}, \alpha_{0}\right)-\gamma\left(\alpha_{0}\right) y_{\delta} z \mathbf{1}_{\{|z|<1\}}(z)\right. \\
\left.\left.\quad \times\left(\frac{2}{\delta}\left(x_{\delta}-y_{\delta}\right)-2 \eta e^{(T-t)} y_{\delta}\right)\right) v(d z), v_{2}\left(t_{\delta}, y_{\delta}, \alpha_{0}\right)-\left(y_{\delta}-a\right)\right] \geq 0 .
\end{aligned}
$$

Therefore, we have

$$
v_{2}\left(t_{\delta}, y_{\delta}, \alpha_{0}\right)-\left(y_{\delta}-a\right) \geq 0
$$


It comes that

$$
v_{1}\left(t_{\delta}, x_{\delta}, \alpha_{0}\right)-v_{2}\left(t_{\delta}, y_{\delta}, \alpha_{0}\right)-\left(x_{\delta}-y_{\delta}\right) \leq 0
$$

Letting $\delta \rightarrow 0$, we obtain

$$
v_{1}\left(t_{0}, x_{0}, \alpha_{0}\right)-v_{2}\left(t_{0}, x_{0}, \alpha_{0}\right) \leq 0
$$

Note that the function $\Phi$ reaches its maximum at $\left(t_{\delta}, x_{\delta}, y_{\delta}, \alpha_{0}\right)$. It follows that for all $x \in \mathbb{R}$, $t \in[s, T]$, and $i \in \mathcal{M}$, we have

$$
\begin{aligned}
v_{1}(t, x, i)-v_{2}(t, x, i)-2 \eta e^{(T-t)} x^{2}=\Phi(x, x, i) \leq & \Phi\left(t_{\delta}, x_{\delta}, y_{\delta}, \alpha_{0}\right) \\
\leq & v_{1}\left(t_{\delta}, x_{\delta}, \alpha_{0}\right)-v_{2}\left(t_{\delta}, y_{\delta}, \alpha_{0}\right) \\
& -\eta e^{\left(T-t_{\delta}\right)}\left(x_{\delta}^{2}+y_{\delta}^{2}\right) .
\end{aligned}
$$

Again letting $\delta \rightarrow 0$ and using (A.61), we obtain

$$
v_{1}(t, x, i)-v_{2}(t, x, i)-2 \eta e^{(T-t)} x^{2} \leq v_{1}\left(t_{0}, x_{0}, \alpha_{0}\right)-v_{2}\left(t_{0}, x_{0}, \alpha_{0}\right)-2 \eta e^{\left(T-t_{0}\right)}\left(x_{0}\right)^{2} \leq 0 .
$$

so, we have

$$
v_{1}(, x, i)-v_{2}(t, x, i) \leq 2 \eta e^{(T-t)} x^{2} .
$$

Second of all, let assume that

$$
\begin{aligned}
& r v_{1}\left(t_{\delta}, x_{\delta}, \alpha_{0}\right)-b_{1 \delta}-\frac{1}{2} x_{\delta}^{2} \sigma^{2}\left(\alpha_{0}\right) X_{\delta}-x_{\delta} \mu\left(\alpha_{0}\right)\left(\frac{2}{\delta}\left(x_{\delta}-y_{\delta}\right)+2 \eta e^{(T-t)} x_{\delta}\right)-Q f\left(s, x_{\delta}, \cdot\right)\left(\alpha_{0}\right) \\
& -\int_{\mathbb{R}}\left(v_{1}\left(t_{\delta}, x_{\delta}+\gamma\left(\alpha_{0}\right) x_{\delta} z\right)-v_{1}\left(t_{\delta}, x_{\delta}\right)-\gamma\left(\alpha_{0}\right) x_{\delta} z \mathbf{1}_{\{|z|<1\}}(z)\left(\frac{2}{\delta}\left(x_{\delta}-y_{\delta}\right)+2 \eta e^{(T-t)} x_{\delta}\right)\right) \\
& \quad \times v(d z) \leq 0,
\end{aligned}
$$

and, moreover, we have

$$
\begin{aligned}
& r v_{2}\left(t_{\delta}, y_{\delta}, \alpha_{0}\right)-b_{2 \delta}-\frac{1}{2} y_{\delta}^{2} \sigma^{2}\left(\alpha_{0}\right) Y_{\delta}-y_{\delta} \mu\left(\alpha_{0}\right)\left(\frac{2}{\delta}\left(x_{\delta}-y_{\delta}\right)-2 \eta e^{\left(T-t_{\delta}\right)} y_{\delta}\right)-Q v_{2}\left(t_{\delta}, y_{\delta}, \cdot\right)\left(\alpha_{0}\right) \\
& -\int_{\mathbb{R}}\left(v_{2}\left(t_{\delta}, y_{\delta}+\gamma\left(\alpha_{0}\right) y_{\delta} z, \alpha_{0}\right)-v_{2}\left(t_{\delta}, y_{\delta}, \alpha_{0}\right)-\gamma\left(\alpha_{0}\right) y_{\delta} z \mathbf{1}_{\{|z|<1\}}(z)\right. \\
& \left.\quad \times\left(\frac{2}{\delta}\left(x_{\delta}-y_{\delta}\right)-2 \eta e^{(T-t)} y_{\delta}\right)\right) v(d z) \geq 0 .
\end{aligned}
$$


International Journal of Mathematics and Mathematical Sciences

Let us use the operator $\mathcal{F}(x, u, \beta, M)$ defined on the Lemma 3.9; thus,

$$
\begin{aligned}
\mathcal{F}(x, v, \beta, M)= & -\frac{1}{2} x^{2} \sigma^{2}\left(\alpha_{0}\right) M-x \mu\left(\alpha_{0}\right) \beta-Q v\left(t_{\delta}, x, \cdot\right)\left(\alpha_{0}\right) \\
& -\int_{\mathbb{R}}\left(v\left(t_{\delta}, x+\gamma\left(\alpha_{0}\right) x z, \alpha_{0}\right)-v\left(t_{\delta}, x, \alpha_{0}\right)-\gamma\left(\alpha_{0}\right) x z \beta\right) v(d z) .
\end{aligned}
$$

Using the operator $\mathcal{F}$, thus (A.65) becomes

$$
r v_{1}\left(t_{\delta}, x_{\delta}, \alpha_{0}\right)+\mathcal{F}\left(x_{\delta}, v_{1}, \frac{2}{\delta}\left(x_{\delta}-y_{\delta}\right)+2 x_{\delta} \eta e^{\left(T-t_{\delta}\right)}, X_{\delta}\right)-b_{1 \delta} \leq 0
$$

and (A.66) becomes

$$
r v_{2}\left(t_{\delta}, y_{\delta}, a_{0}\right)+\mp\left(y_{\delta}, v_{2}, \frac{2}{\delta}\left(x_{\delta}-y_{\delta}\right)-2 y_{\delta} \eta e^{\left(T-t_{\delta}\right)}, Y_{\delta}\right)-b_{2 \delta} \geq 0
$$

Combining the last two inequalities, we obtain

$$
\begin{aligned}
r\left(v_{1}\left(t_{\delta}, x_{\delta}, \alpha_{0}\right)-v_{2}\left(t_{\delta}, y_{\delta}, \alpha_{0}\right)\right) \leq & \mathcal{F}\left(y_{\delta}, v_{2}, \frac{2}{\delta}\left(x_{\delta}-y_{\delta}\right)-2 y_{\delta} \eta e^{\left(T-t_{\delta}\right)}, Y_{\delta}\right) \\
& -\mathcal{F}\left(x_{\delta}, v_{1}, \frac{2}{\delta}\left(x_{\delta}-y_{\delta}\right)+2 x_{\delta} \eta e^{\left(T-t_{\delta}\right)}, X_{\delta}\right)+b_{2 \delta}-b_{1 \delta}
\end{aligned}
$$

From Lemma 3.9, there exists a constant $C>0$ such that

$$
\begin{gathered}
\mathcal{F}\left(y_{\delta}, v_{2}, \frac{2}{\delta}\left(x_{\delta}-y_{\delta}\right)-2 y_{\delta} \eta e^{\left(T-t_{\delta}\right)}, Y_{\delta}\right)-\mathcal{F}\left(x_{\delta}, v_{1}, \frac{2}{\delta}\left(x_{\delta}-y_{\delta}\right)+2 x_{\delta} \eta e^{\left(T-t_{\delta}\right)}, X_{\delta}\right) \\
\leq C\left(\frac{2}{\delta}\left|x_{\delta}-y_{\delta}\right|^{2}+2 \eta e^{\left(T-t_{\delta}\right)}\left(x_{\delta}^{2}+y_{\delta}^{2}\right)+\left(x_{\delta}^{2} X_{\delta}-y_{\delta}^{2} Y_{\delta}\right)\right)+Q v\left(t_{\delta}, x, \cdot\right)\left(\alpha_{0}\right) \\
-Q w\left(t_{\delta}, y_{1} \cdot\right)\left(\alpha_{0}\right)+\int_{\mathbb{R}}\left(\left[v\left(t_{\delta}, x+\gamma\left(\alpha_{0}\right) x z, \alpha_{0}\right)-w\left(t_{\delta}, y+\gamma\left(\alpha_{0}\right) y z, \alpha_{0}\right)\right]\right. \\
\left.-\left[v\left(t_{\delta}, x, \alpha_{0}\right)-w\left(t_{\delta}, y, \alpha_{0}\right)\right]\right) v(d z) .
\end{gathered}
$$

Recall that $Q v(t, x, \cdot)(i)=\sum_{j \neq i} v(t, x, j)-v(t, x, i)$, so we have

$$
\begin{aligned}
Q v_{1}\left(t_{\delta}, x_{\delta}, \cdot\right)\left(\alpha_{0}\right)-Q v_{2}\left(t_{\delta}, y_{\delta}, \cdot\right)\left(\alpha_{0}\right)=\sum_{i \neq \alpha_{\delta}} & {\left[v_{1}\left(t_{\delta}, x_{\delta}, i\right)-v_{1}\left(t_{\delta}, x_{\delta}, \alpha_{0}\right)\right] } \\
- & {\left[v_{2}\left(t_{\delta}, y_{\delta}, i\right)-v_{2}\left(t_{\delta}, y_{\delta}, \alpha_{0}\right)\right] . }
\end{aligned}
$$


Since $\Phi$ attains its maximum at $\left(t_{\delta}, x_{\delta}, y_{\delta}, \alpha_{0}\right)$, we have $\Phi\left(t_{\delta}, x_{\delta}, y_{\delta}, i\right) \leq \Phi\left(t_{\delta}, x_{\delta}, y_{\delta}, \alpha_{0}\right)$; therefore

$$
\begin{aligned}
& v_{1}\left(t_{\delta}, x_{\delta}, i\right)-v_{2}\left(t_{\delta}, y_{\delta}, i\right)-\frac{1}{\delta}\left|x_{\delta}-y_{\delta}\right|^{2}+\eta e^{T-t_{\delta}}\left(\left|x_{\delta}\right|^{2}+\left|y_{\delta}\right|^{2}\right) \\
& \quad \leq v_{1}\left(t_{\delta}, x_{\delta}, \alpha_{0}\right)-v_{2}\left(t_{\delta}, y_{\delta}, \alpha_{0}\right)-\frac{1}{\delta}\left|x_{\delta}-y_{\delta}\right|^{2}-\eta e^{T-t_{\delta}}\left(\left|x_{\delta}\right|^{2}+\left|y_{\delta}\right|^{2}\right)
\end{aligned}
$$

Thus, we have

$$
v_{1}\left(t_{\delta}, x_{\delta}, i\right)-v_{2}\left(t_{\delta}, y_{\delta}, i\right) \leq v_{1}\left(t_{\delta}, x_{\delta}, \alpha_{0}\right)-v_{2}\left(t_{\delta}, y_{\delta}, \alpha_{0}\right)
$$

Consequently, from (A.74) it comes that (A.72) implies

$$
Q v_{1}\left(t_{\delta}, x_{\delta}, \cdot\right)\left(\alpha_{0}\right)-Q v_{2}\left(t_{\delta}, y_{\delta}, \cdot\right)\left(\alpha_{0}\right) \leq 0
$$

Note that from (3.11), we have

$$
b_{1 \delta}-b_{2 \delta}=\frac{\partial \phi\left(t_{\delta}, x_{\delta}, y_{\delta}\right)}{\partial t}=\eta e^{\left(T-t_{\delta}\right)}\left(\left(x_{\delta}\right)^{2}+\left(y_{\delta}\right)^{2}\right)
$$

Therefore, we have

$$
\begin{aligned}
r\left(v_{1}\left(t_{\delta}, x_{\delta}, \alpha_{0}\right)-v_{2}\left(t_{\delta}, y_{\delta}, \alpha_{0}\right)\right) \leq & C\left(\frac{2}{\delta}\left|x_{\delta}-y_{\delta}\right|^{2}+2 \eta e^{\left(T-t_{\delta}\right)}\left(x_{\delta}^{2}+y_{\delta}^{2}\right)+\left(x_{\delta}^{2} X_{\delta}-y_{\delta}^{2} Y_{\delta}\right)\right) \\
& +\int_{\mathbb{R}}\left(\left[v_{1}\left(t_{\delta}, x_{\delta}+\gamma\left(\alpha_{0}\right) x_{\delta} z, \alpha_{0}\right)-v_{2}\left(t_{\delta}, y_{\delta}+\gamma\left(\alpha_{0}\right) y_{\delta} z, \alpha_{0}\right)\right]\right. \\
- & {\left.\left[v_{1}\left(t_{\delta}, x_{\delta}, \alpha_{0}\right)-v_{2}\left(t_{\delta}, y_{\delta}, \alpha_{0}\right)\right]\right) v(d z) . }
\end{aligned}
$$

Similarly, for any $z \in \mathbb{R}$, we have

$$
\Phi\left(t_{\delta}, x_{\delta}+\gamma\left(\alpha_{0}\right) x_{\delta} z, y_{\delta}+\gamma\left(\alpha_{0}\right) y_{\delta} z, \alpha_{0}\right) \leq \Phi\left(t_{\delta}, x_{\delta}, y_{\delta}, \alpha_{0}\right)
$$


International Journal of Mathematics and Mathematical Sciences consequently, we derive that

$$
\begin{gathered}
\int_{\mathbb{R}}\left[v_{1}\left(t_{\delta}, x_{\delta}+\gamma\left(\alpha_{0}\right) x_{\delta} z, \alpha_{0}\right)-v_{2}\left(t_{\delta}, y_{\delta}+\gamma\left(\alpha_{0}\right) y_{\delta} z, \alpha_{0}\right)\right] v(d z) \\
\leq \int_{\mathbb{R}}\left[v_{1}\left(t_{\delta}, x_{\delta}, \alpha_{\delta}\right)-v_{2}\left(t_{\delta}, y_{\delta}, \alpha_{\delta}\right)+\frac{1}{\delta}\left|\left(x_{\delta}+\gamma\left(\alpha_{0}\right) x_{\delta} z\right)-\left(y_{\delta}+\gamma\left(\alpha_{0}\right) y_{\delta} z\right)\right|^{2}\right. \\
\left.\quad+\eta e^{T-t_{\delta}}\left(\left|x_{\delta}+\gamma\left(\alpha_{0}\right) x_{\delta} z\right|^{2}-\left|y_{\delta}+\gamma\left(\alpha_{0}\right) y_{\delta} z\right|^{2}\right)\right] v(d z) \\
\leq \int_{\mathbb{R}}\left[v_{1}\left(t_{\delta}, x_{\delta}, \alpha_{0}\right)-v_{2}\left(t_{\delta}, y_{\delta}, \alpha_{0}\right)+\frac{1}{\delta}\left(\left|x_{\delta}-y_{\delta}\right|^{2}+\left|\gamma\left(\alpha_{0}\right) x_{\delta} z-\gamma\left(\alpha_{0}\right) y_{\delta} z\right|^{2}\right)\right. \\
\left.\quad+\eta e^{T-t_{\delta}}\left(\left|x_{\delta}\right|^{2}+\left|\gamma\left(\alpha_{0}\right) x_{\delta} z\right|^{2}+\left|y_{\delta}\right|^{2}+\left|\gamma\left(\alpha_{0}\right) y_{\delta} z\right|^{2}\right)\right] v(d z) .
\end{gathered}
$$

Therefore, using (2.3), we obtain

$$
\begin{aligned}
& \int_{\mathbb{R}}( {\left[v_{1}\left(t_{\delta}, x_{\delta}+\gamma\left(\alpha_{0}\right) x_{\delta} z, \alpha_{0}\right)-v_{2}\left(t_{\delta}, y_{\delta}+\gamma\left(\alpha_{0}\right) y_{\delta} z, \alpha_{0}\right)\right] } \\
&- {\left.\left[v_{1}\left(t_{\delta}, x_{\delta}, \alpha_{0}\right)-v_{2}\left(t_{\delta}, y_{\delta}, \alpha_{0}\right)\right]\right) v(d z) } \\
& \leq \int_{\mathbb{R}} {\left[\frac{1}{\delta}\left(\left|x_{\delta}-y_{\delta}\right|^{2}+\left|\gamma\left(\alpha_{0}\right) x_{\delta} z-\gamma\left(\alpha_{0}\right) y_{\delta} z\right|^{2}\right)\right.} \\
&\left.+\eta e^{T-t_{\delta}}\left(\left|x_{\delta}\right|^{2}+\left|\gamma\left(\alpha_{0}\right) x_{\delta} z\right|^{2}+\left|y_{\delta}\right|^{2}+\left|\gamma\left(\alpha_{0}\right) y_{\delta} z\right|^{2}\right)\right] v(d z) \\
& \leq C\left(\frac{1}{\delta}\left|x_{\delta}-y_{\delta}\right|^{2}+\eta e^{T-t_{\delta}}\left(1+\left|x_{\delta}\right|^{2}+\left|y_{\delta}\right|^{2}\right)\right),
\end{aligned}
$$

for some constant $C>0$. Taking into account (A.75) and (A.80), thus (A.77) becomes

$$
r\left(v_{1}\left(t_{\delta}, x_{\delta}, \alpha_{0}\right)-v_{2}\left(t_{\delta}, y_{\delta}, \alpha_{0}\right)\right) \leq C\left(\frac{2}{\delta}\left|x_{\delta}-y_{\delta}\right|^{2}+2 \eta e^{\left(T-t_{\delta}\right)}\left(x_{\delta}^{2}+y_{\delta}^{2}\right)+\left|x_{\delta}^{2} X_{\delta}-y_{\delta}^{2} Y_{\delta}\right|\right)
$$

We know from the Maximum principle that

$$
\begin{aligned}
-\left(\frac{1}{\epsilon}+\left\|D_{(x, y)}^{2} \phi\left(t_{\delta}, x_{\delta}, y_{\delta}\right)\right\|\right) I & \leq\left(\begin{array}{cc}
X_{\delta} & 0 \\
0 & -Y_{\delta}
\end{array}\right) \\
& \leq D_{(x, y)}^{2} \phi\left(t_{\delta}, x_{\delta}, y_{\delta}\right)+\epsilon\left(D_{(x, y)}^{2} \phi\left(t_{\delta}, x_{\delta}, y_{\delta}\right)\right)^{2} .
\end{aligned}
$$


Moreover,

$$
\begin{aligned}
D_{(x, y)}^{2} \phi\left(t_{\delta}, x_{\delta}, y_{\delta}\right) & =\frac{2}{\delta}\left(\begin{array}{cc}
1 & -1 \\
-1 & 1
\end{array}\right)+2 \eta e^{\left(T-t_{\delta}\right)}\left(\begin{array}{ll}
1 & 0 \\
0 & 1
\end{array}\right), \\
\left(D_{(x, y)}^{2} \phi\left(t_{\delta}, x_{\delta}, y_{\delta}\right)\right)^{2} & =\frac{8}{\delta^{2}}\left(\begin{array}{cc}
1 & -1 \\
-1 & 1
\end{array}\right)+\frac{8 \eta e^{\left(T-t_{\delta}\right)}}{\delta}\left(\begin{array}{cc}
1 & -1 \\
-1 & 1
\end{array}\right)+4 \eta^{2} e^{2\left(T-t_{\delta}\right)}\left(\begin{array}{ll}
1 & 0 \\
0 & 1
\end{array}\right) \\
& =\frac{8+8 \eta \delta e^{\left(T-t_{\delta}\right)}}{\delta^{2}}\left(\begin{array}{cc}
1 & -1 \\
-1 & 1
\end{array}\right)+4 \eta^{2} e^{2\left(T-t_{\delta}\right)}\left(\begin{array}{ll}
1 & 0 \\
0 & 1
\end{array}\right) .
\end{aligned}
$$

Note that

$$
\begin{aligned}
&\left(x_{\delta}\right)^{2} X_{\delta}-\left(y_{\delta}\right)^{2} Y_{\delta}=\left(x_{\delta}, y_{\delta}\right)\left(\begin{array}{cc}
X_{\delta} & 0 \\
0 & -Y_{\delta}
\end{array}\right)\left(\begin{array}{l}
x_{\delta} \\
y_{\delta}
\end{array}\right) \\
& \leq\left(x_{\delta}, y_{\delta}\right)\left[\frac{2}{\delta}\left(\begin{array}{cc}
1 & -1 \\
-1 & 1
\end{array}\right)+\left(2 \eta e^{\left(T-t_{\delta}\right)}+4 \epsilon \eta^{2} e^{2\left(T-t_{\delta}\right)}\right)\left(\begin{array}{ll}
1 & 0 \\
0 & 1
\end{array}\right)\right. \\
&\left.+\epsilon \frac{8+8 \eta \delta e^{\left(T-t_{\delta}\right)}}{\delta^{2}}\left(\begin{array}{cc}
1 & -1 \\
-1 & 1
\end{array}\right)\right]\left(\begin{array}{l}
x_{\delta} \\
y_{\delta}
\end{array}\right) .
\end{aligned}
$$

Letting $\eta \rightarrow 0$, we obtain

$$
\left(x_{\delta}\right)^{2} X_{\delta}-\left(y_{\delta}\right)^{2} Y_{\delta} \leq\left(x_{\delta}, y_{\delta}\right)\left[\left(\frac{2}{\delta}+\epsilon \frac{8}{\delta^{2}}\right)\left(\begin{array}{cc}
1 & -1 \\
-1 & 1
\end{array}\right)\right]\left(\begin{array}{l}
x_{\delta} \\
y_{\delta}
\end{array}\right) .
$$

Take $\epsilon=\delta / 4$, this leads to

$$
\left(x_{\delta}\right)^{2} X_{\delta}-\left(y_{\delta}\right)^{2} Y_{\delta} \leq\left(x_{\delta}, y_{\delta}\right)\left[\frac{4}{\delta}\left(\begin{array}{cc}
1 & -1 \\
-1 & 1
\end{array}\right)\right]\left(\begin{array}{l}
x_{\delta} \\
y_{\delta}
\end{array}\right)=\frac{4}{\delta}\left(x_{\delta}-y_{\delta}\right)^{2} .
$$

Using (A.50), we obtain

$$
\begin{aligned}
\limsup _{\delta \downarrow 0}\left(x_{\delta}\right)^{2} X_{\delta}-\left(y_{\delta}\right)^{2} Y_{\delta} & \leq \underset{\delta \downarrow 0}{\limsup }\left(x_{\delta}, y_{\delta}\right)\left[\frac{4}{\delta}\left(\begin{array}{cc}
1 & -1 \\
-1 & 1
\end{array}\right)\right]\left(\begin{array}{l}
x_{\delta} \\
y_{\delta}
\end{array}\right) \\
& =\underset{\delta \downarrow 0}{\limsup } \frac{4}{\delta}\left(x_{\delta}-y_{\delta}\right)^{2}=0 .
\end{aligned}
$$

Letting $\eta \rightarrow 0$ in (A.81), we have

$$
r\left(v_{1}\left(t_{\delta}, x_{\delta}, \alpha_{0}\right)-v_{2}\left(t_{\delta}, y_{\delta}, \alpha_{0}\right)\right) \leq C\left(\frac{2}{\delta}\left|x_{\delta}-y_{\delta}\right|^{2}+\left(x_{\delta}^{2} X_{\delta}-y_{\delta}^{2} Y_{\delta}\right)\right)
$$


and taking the lim sup as $\delta$ goes to zero and using (A.87), we obtain

$$
r\left(v_{1}\left(t_{0}, x_{0}, \alpha_{0}\right)-v_{2}\left(t_{0}, x_{0}, \alpha_{0}\right)\right) \leq 0
$$

Recall that $\left(t_{\delta}, x_{\delta}, y_{\delta}, \alpha_{0}\right)$ is maximum of $\Phi$. Then, for all $x \in \mathbb{R}, t \in[s, T]$, and for all $i \in \mathcal{M}$, we have

$$
\Phi(t, x, x, i) \leq \Phi\left(t_{\delta}, x_{\delta}, y_{\delta}, \alpha_{0}\right)
$$

It comes that

$$
v_{1}(t, x, i)-v_{2}(t, x, i)-2 \eta e^{(T-t)} x^{2} \leq v_{1}\left(t_{\delta}, x_{\delta}, \alpha_{0}\right)-v_{2}\left(t_{\delta}, y_{\delta}, \alpha_{0}\right)-2 \eta e^{\left(T-t_{\delta}\right)}\left(x_{\delta}^{2}+y_{\delta}^{2}\right) .
$$

Letting $\delta \rightarrow 0$, we obtain

$$
v_{1}(t, x, i)-v_{2}(t, x, i)-2 \eta e^{(T-t)} x^{2} \leq v_{1}\left(t_{0}, x_{0}, \alpha_{0}\right)-v_{2}\left(t_{0}, x_{0}, \alpha_{0}\right)-2 \eta e^{(T-t)} x_{0}^{2} .
$$

Using (A.89), we have

$$
v_{1}\left(t_{0}, x_{0}, \alpha_{0}\right)-v_{2}\left(t_{0}, x_{0}, \alpha_{0}\right) \leq 0
$$

Therefore, using (A.92), we conclude that

$$
v_{1}(t, x, i)-v_{2}(t, x, i)-2 \eta e^{(T-t)} x^{2} \leq v_{1}\left(t_{0}, x_{0}, \alpha_{0}\right)-v_{2}\left(t_{0}, x_{0}, \alpha_{0}\right)-2 \eta e^{\left(T-t_{0}\right)} x_{0}^{2} \leq 0 .
$$

Letting $\eta \rightarrow 0$ in (A.64) and the previous inequality, we have

$$
v_{1}(t, x, i) \leq v_{2}(t, x, i)
$$

This completes the proof of the theorem.

\section{Acknowledgment}

The author is grateful to the editor and the referee for their helpful suggestions and comments.

\section{References}

[1] Q. Zhang, "Stock trading: an optimal selling rule," SIAM Journal on Control and Optimization, vol. 40, no. 1, pp. 64-87, 2001.

[2] M. Pemy and Q. Zhang, "Optimal stock liquidation in a regime switching model with finite time horizon," Journal of Mathematical Analysis and Applications, vol. 321, no. 2, pp. 537-552, 2006. 
[3] H. P. McKean, "A free boundary problem for the heat equation arising from a problem in mathematical economics," Industrial Management Review, vol. 60, pp. 32-39, 1960.

[4] P. A. Samuelson, "Rational theory of warrant pricing," Industrial Management Review, vol. 6, pp. 13-32, 1995.

[5] B. ØKsendal, Stochastic Differential Equations, Springer, New York, NY, USA, 1998.

[6] X. Guo and Q. Zhang, "Optimal selling rules in a regime switching model," Institute of Electrical and Electronics Engineers Transactions on Automatic Control, vol. 50, no. 9, pp. 1450-1455, 2005.

[7] N. V. Krylov, Controlled Diffusion Processes, vol. 14 of Applications of Mathematics, Springer, Berlin, Germany, 1980, Translated from the Russian by A. B. Arie.

[8] H. Pham, "Optimal stopping of controlled jump diffusion processes: a viscosity solution approach," Journal of Mathematical Systems, Estimation, and Control, vol. 8, no. 1, pp. 1-27, 1998.

[9] M. Pemy, Option pricing under regime witching, Ph.D. thesis, University of Georgia, Athens, Ga, USA, 2005.

[10] M. G. Crandall, H. Ishii, and P.-L. Lions, “User's guide to viscosity solutions of second order partial differential equations," Bulletin of the American Mathematical Society, vol. 27, no. 1, pp. 1-67, 1992.

[11] G. G. Yin and Q. Zhang, Continuous-Time Markov Chains and Applications: A Singular Perturbation Approach, vol. 37, Springer, New York, NY, USA, 1998.

[12] W. H. Fleming and H. M. Soner, Controlled Markov Processes and Viscosity Solutions, vol. 25 of Stochastic Modelling and Applied Probability, Springer, New York, NY, USA, 2nd edition, 2006. 


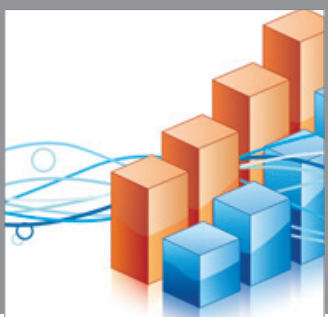

Advances in

Operations Research

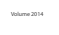

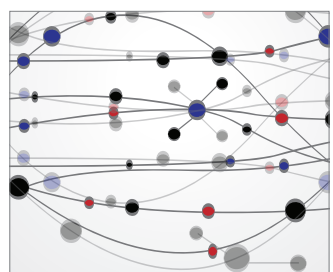

\section{The Scientific} World Journal
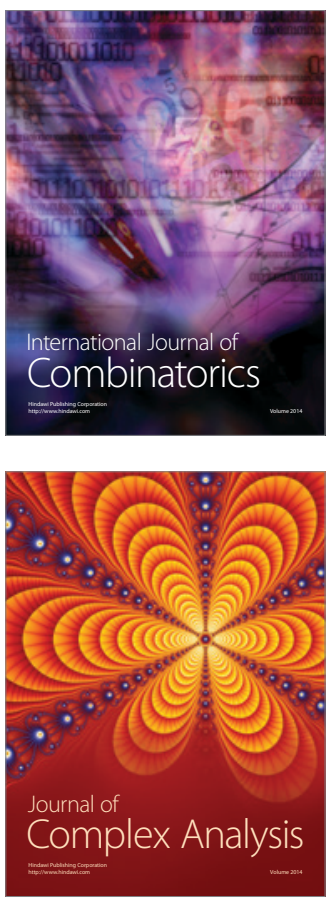

International Journal of

Mathematics and

Mathematical

Sciences
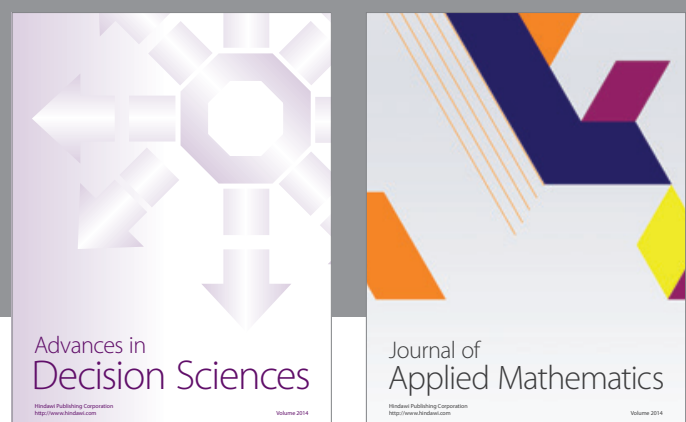

Journal of

Applied Mathematics
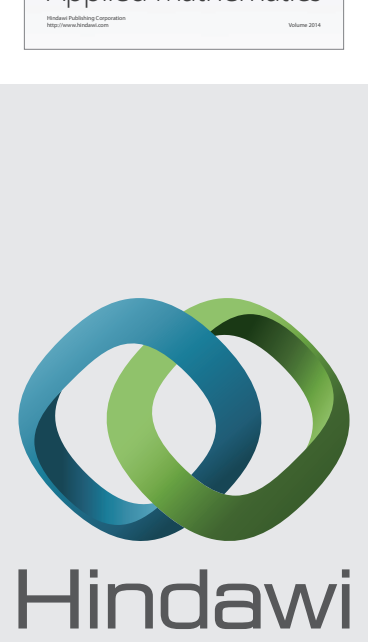

Submit your manuscripts at http://www.hindawi.com
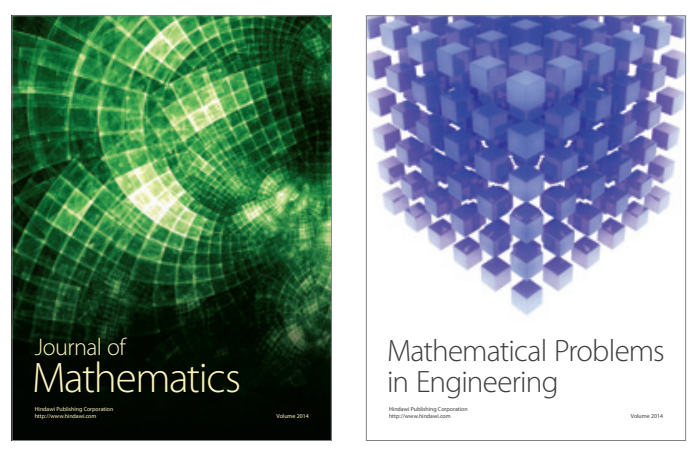

Mathematical Problems in Engineering
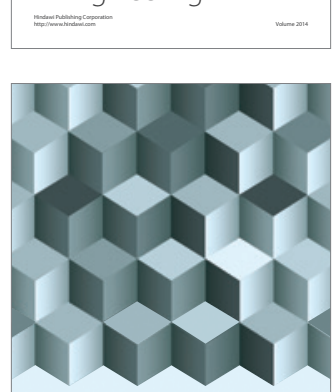

Journal of

Function Spaces
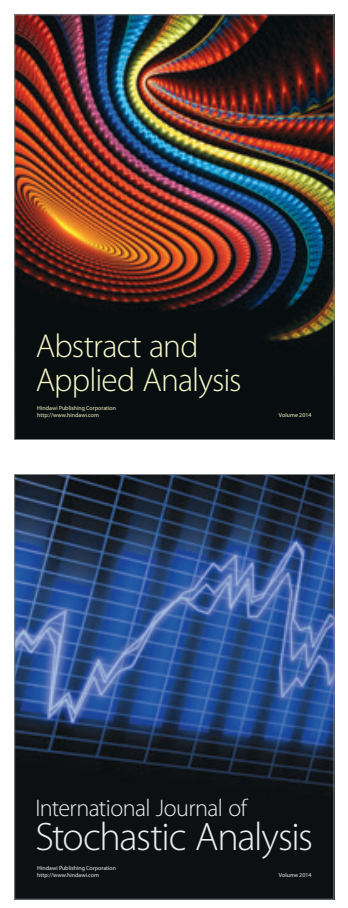

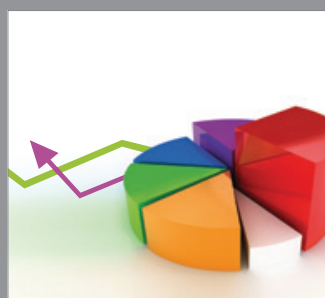

ournal of

Probability and Statistics

Promensencen
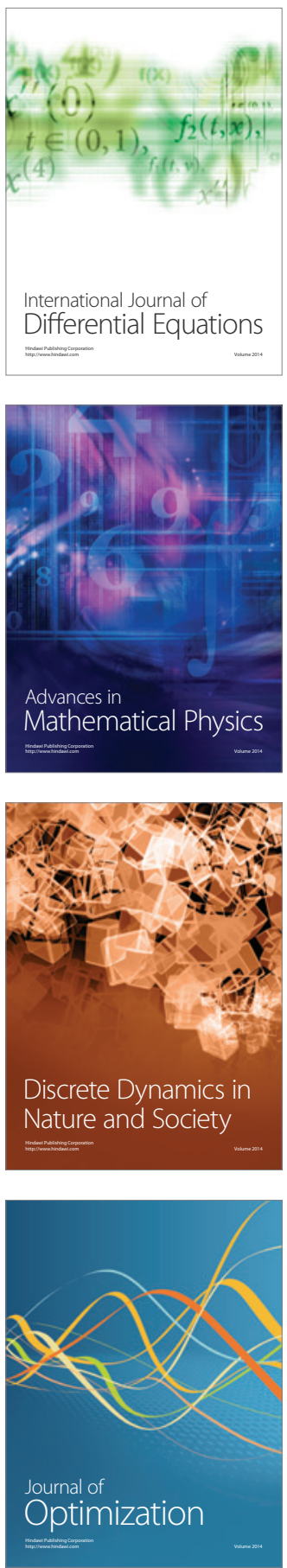